Article

\title{
Characterisation of Mediterranean Grape Pomace Seed and Skin Extracts: Polyphenolic Content and Antioxidant Activity
}

\author{
Isabelle Ky ${ }^{1,2}$ and Pierre-Louis Teissedre ${ }^{1,2, *}$ \\ 1 Univ Bordeaux, ISVV, EA 4577 Enologie, 210 Chemin de Leysotte, \\ Villenave d'Ornon F-33140, France \\ 2 INRA, ISVV, USC 1366 CEnologie, 210 Chemin de Leysotte, Villenave d'Ornon F-33140, France \\ * Author to whom correspondence should be addressed; E-Mail: p.teissedre@u-bordeaux2.fr; \\ Tel.: +33-0-557-575-850; Fax: +33-0-557-575-813.
}

Academic Editor: Maurizio Battino

Received: 25 November 2014 / Accepted: 19 January 2015 / Published: 29 January 2015

\begin{abstract}
Grape pomace seeds and skins from different Mediterranean varieties (Grenache [GRE], Syrah [SYR], Carignan [CAR], Mourvèdre [MOU] and Alicante [ALI]) were extracted using water and water/ethanol $70 \%$ in order to develop edible extracts (an aqueous extract [EAQ] and a 70\% hydro-alcoholic extract [EA70]) for potential use in nutraceutical or cosmetic formulations. In this study, global content (total polyphenols, total anthocyanins and total tannins), flavan-3-ols and anthocyanins were assessed using HPLC-UV-Fluo-MS ${ }^{\mathrm{n}}$. In addition, extract potential was evaluated by four different assays: Oxygen Radical Absorbance Capacity (ORAC), Ferric Reducing Antioxidant Potential assay (FRAP), Trolox equivalent antioxidant capacity (TEAC) or ABTS assay and 2,2-diphenyl-1-picrylhydrazyl (DPPH) radical scavenging assay. As expected, seed pomace extracts contained higher amounts of polyphenols then skin pomace extracts. Indeed, seeds from Syrah contained a particularly important amount of total polyphenols and tannins in both type of extract (up to $215.84 \pm 1.47 \mathrm{mg}$ of gallic acid equivalent [GAE]/g dry weight (DW) and $455.42 \pm 1.84 \mathrm{mg} / \mathrm{g}$ DW, respectively). These extracts also expressed the highest antioxidant potential with every test. For skins, the maximum total phenolic was found in Alicante EAQ $(196.71 \pm 0.37 \mathrm{mg} \mathrm{GAE} / \mathrm{g} \mathrm{DW})$ and in Syrah EA70 $(224.92 \pm 0.18 \mathrm{mg} \mathrm{GAE} / \mathrm{g}$ DW). Results obtained in this article constitute a useful tool for the pre-selection of grape pomace seed and skin extracts for nutraceutical purposes.
\end{abstract}


Keywords: grape pomaces; Mediterranean varieties; phenolic compounds; flavan-3-ols; anthocyanins; antioxidant activity

\section{Introduction}

Phenolic compounds are currently receiving much attention because of their beneficial health effects related to their ability to protect against oxidative cell damage when antioxidant and pro-oxidant imbalances occur. Indeed, reactive species play both a beneficial and toxic role and the balance between them has to be maintained [1]. To prevent an overload of free radicals and peroxides, aerobic organisms use a sophisticated defense system which operates in both the intra- and extracellular aqueous phases and in membranes. Antioxidant defense strategies are committed to prevent the oxidative attack in its early moments by the formation of priming radicals as well as during the initiation and chain propagation stages under stress condition or during aging processes, excessive levels of reactive species may interrupt regular processes. The exposure of tissues to oxidative stress could then generate a cascade of degenerative processes [2].

Clinical and nutritional epidemiological studies have shown an inverse correlation between the consumption of polyphenol-enriched diets and a reduced risk of cardiovascular diseases, along with their ensuing complications and related mortality [3-5]. Actually, polyphenolic compounds serve as reducing agents in many biological systems by donating hydrogen, quenching singlet oxygen, acting as chelators and by trapping free radicals. Moreover, these antioxidant activities can help to limit oxidation of nucleic acids, proteins, lipids, which may initiate degenerative diseases such as neuro-degenerative disease, cancer, heart disease, chronic inflammation, dermal disorders and aging [6-8].

Vitis vinifera grapes, one of the most cultivated fruit crops in the world with an annual production of $\sim 64$ million metric tons in 2010 [9], are known to be rich in polyphenols. Each year, the wine making industry produces a substantial amount of grape by-products called pomaces which account for about $20 \%$ of the weight of the grapes used to make wine [10,11]. The high polyphenol content of grapes and the far from complete extraction of grape polyphenols during vinification, which typically reaches only ca. $30 \%-40 \%$, depending on grape varieties, vineyard location and technological parameters of wine making including destemming, crushing, maceration and pressing $[12,13]$ make grape pomace potentially a very abundant and relatively inexpensive source of a wide range of polyphenols, including monomeric and oligomeric proanthocyanidins and a diversity of anthocyanin glycosides [14-16]. Significant efforts have been devoted over the past decade to explore the potential of using grape pomace to produce functional food ingredients, such as natural antioxidants for nutrition fortification and food preservation [17]. Other alternative potential commercial uses of grape pomaces that have been advocated include food colorings and ingredients [18-20], dietary fibers [10,21], phytochemical products [22] and dietary supplements for disease prevention [23]. Therefore, the aim of this study was to analyse the potential in this respect of grape by-products from important Rhône Valley red wine cultivars: Grenache, Syrah, Carignan, Mourvèdre and Alicante. Seeds and skins were extracted using water and water/ethanol $70 \%$ in order to develop two types of edible extracts: aqueous extracts (EAQ) and hydro-alcoholic $70 \%$ extracts (EA70). We reported herein the total polyphenol, total anthocyanin and total tannin contents as 
well as the determination and quantification of flavan-3-ols (monomers, dimers) and anthocyanins using HPLC with absorbance, fluorescence and mass detection. Moreover, the antioxidant capacity of pomace extracts was assessed using four antioxidant assays $\left(\mathrm{ABTS}^{+}\right.$, DPPH, FRAP and ORAC). The data may contribute to the selection of suitable seed and skin pomace extracts for the development of antioxidant- and polyphenolic-rich nutraceuticals.

\section{Results and Discussion}

\subsection{Total Phenol, Total Tannin and Total Anthocyanin Analysis of Grape Pomace Seed and} Skin Extracts

Seeds of Grenache (1st location), Syrah and Carignan [GRE1, SYR1 and CAR] and skins from Grenache (2nd location), Syrah (two different locations), Carignan, Mourvèdre and Alicante [GRE2, SYR1, SYR2, CAR, MOU and ALI] were extracted using water and 70\% hydro-alcoholic solution, thus giving two types of samples: aqueous samples (EAQ) and 70\% hydro-alcoholic samples (EA70). Aqueous and $70 \%$ hydro-alcoholic extracts were characterized for their overall composition by total phenol content, total tannin and total anthocyanin analysis via Folin-Ciocalteu assay, acidic hydrolysis and $\mathrm{SO}_{2}$ bleaching procedure. Results are presented in Table 1 for seed extracts and Table 2 concerning skin extracts.

Table 1. Total phenol contents, total tannins, total anthocyanins and flavan-3-ol monomers, dimers and trimer characterisation in EAQ and EA70 grape pomace seed extracts.

\begin{tabular}{|c|c|c|c|c|c|c|}
\hline & \multicolumn{3}{|c|}{ Seeds-EAQ } & \multicolumn{3}{|c|}{ Seeds-EA70 } \\
\hline & GRE1 a & SYR1 ${ }^{a}$ & CAR $^{\text {a }}$ & GRE1 a & SYR1 ${ }^{a}$ & CAR $^{\text {a }}$ \\
\hline \multicolumn{7}{|l|}{ Total composition: } \\
\hline TPC & $128.22 \pm 0.37 \mathrm{a}$ & $215.93 \pm 1.17 \mathrm{c}$ & $186.08 \pm 0.28 b$ & $195.66 \pm 1.06 \mathrm{a}$ & $207.38 \pm 2.15 b$ & $215.84 \pm 1.47 \mathrm{~b}$ \\
\hline Total tannins & $157.02 \pm 0.56 \mathrm{a}$ & $266.87 \pm 2.62 b$ & $264.61 \pm 2.39 b$ & $302.86 \pm 4.85 \mathrm{a}$ & $455.42 \pm 1.84 b$ & $423.11 \pm 15.13 b$ \\
\hline Total anthocyanins & $3.98 \pm 0.16 \mathrm{a}$ & $10.55 \pm 0.56 b$ & $11.35 \pm 0.51 b$ & $12.17 \pm 0.51 \mathrm{a}$ & $38.67 \pm 4.34 \mathrm{~b}$ & $57.34 \pm 1.86 b$ \\
\hline \multicolumn{7}{|c|}{ Proanthocyanidins composition: } \\
\hline $\mathbf{C}$ & $2.07 \pm 0.09 \mathrm{a}$ & $5.12 \pm 0.04 b$ & $2.27 \pm 0.00 \mathrm{a}$ & $3.60 \pm 0.02 \mathrm{a}$ & $8.60 \pm 0.00 c$ & $5.28 \pm 0.03 b$ \\
\hline EC & $0.98 \pm 0.04 \mathrm{a}$ & $3.76 \pm 0.03 b$ & $0.94 \pm 0.00 \mathrm{a}$ & $1.46 \pm 0.00 \mathrm{a}$ & $5.24 \pm 0.00 \mathrm{c}$ & $2.02 \pm 0.06 \mathrm{~b}$ \\
\hline$\Sigma$ Monomers & $3.04 \pm 0.09 \mathrm{a}$ & $8.88 \pm 0.00 \mathrm{~b}$ & $3.21 \pm 0.00 \mathrm{a}$ & $5.07 \pm 0.01 \mathrm{a}$ & $13.84 \pm 0.00 \mathrm{c}$ & $7.29 \pm 0.02 b$ \\
\hline $\mathbf{B}_{1}$ & $1.01 \pm 0.08 \mathrm{a}$ & $2.94 \pm 0.01 b$ & $0.87 \pm 0.01 \mathrm{a}$ & $1.68 \pm 0.01 \mathrm{a}$ & $3.53 \pm 0.01 \mathrm{c}$ & $3.06 \pm 0.00 \mathrm{~b}$ \\
\hline $\mathbf{B}_{2}$ & $0.70 \pm 0.01 \mathrm{a}$ & $2.23 \pm 0.02 b$ & $0.68 \pm 0.00 \mathrm{a}$ & $0.84 \pm 0.00 \mathrm{a}$ & $2.16 \pm 0.02 c$ & $1.29 \pm 0.00 b$ \\
\hline $\mathbf{B}_{3}$ & $0.28 \pm 0.02 \mathrm{a}$ & $0.86 \pm 0.00 \mathrm{~b}$ & $0.25 \pm 0.00 \mathrm{a}$ & $0.45 \pm 0.02 \mathrm{a}$ & $0.87 \pm 0.02 \mathrm{c}$ & $0.58 \pm 0.01 b$ \\
\hline $\mathbf{B}_{4}$ & $0.51 \pm 0.01 b$ & $0.85 \pm 0.04 c$ & $0.11 \pm 0.00 \mathrm{a}$ & $\mathrm{Nd}$ & $0.53 \pm 0.01$ & $\mathrm{Nd}$ \\
\hline$\Sigma$ Dimers & $2.50 \pm 0.05 b$ & $6.870 \pm 0.03 c$ & $1.90 \pm 0.01 \mathrm{a}$ & $2.97 \pm 0.00 \mathrm{a}$ & $7.10 \pm 0.03 c$ & $4.92 \pm 0.01 b$ \\
\hline $\mathbf{C}_{1}$ & $0.48 \pm 0.01 \mathrm{a}$ & $2.00 \pm 0.06 b$ & $0.53 \pm 0.01 \mathrm{a}$ & $0.54 \pm 0.00 \mathrm{a}$ & $1.25 \pm 0.03 c$ & $0.83 \pm 0.01 b$ \\
\hline
\end{tabular}

${ }^{a}$ GRE1, Grenache; SYR1, Syrah; CAR, Carignan. In units of mg gallic acid equivalent (GAE)/g DW of seeds for TPC and $\mathrm{mg} / \mathrm{g}$ DW of seeds for total tannins, total anthocyanins and the quantification of proanthocyanidins. Data are expressed as the mean of triplicate \pm standard deviation. TPC, total phenol contents; C, (+)-Catechin; EC, (-)-Epicatechin; $\mathrm{B}_{1}, \mathrm{~B}_{2} ; \mathrm{B}_{3}, \mathrm{~B}_{4}$, Procyanidin dimers $\mathrm{B}_{1}, \mathrm{~B}_{2} ; \mathrm{B}_{3}, \mathrm{~B}_{4} ; \mathrm{C} 1$, procyanidin trimers $\mathrm{C}_{1} . \Sigma$ Monomers, sum of catechin and epicatechin; $\Sigma$ Dimers, sum of $\mathrm{B}_{1}, \mathrm{~B}_{2}, \mathrm{~B}_{3}$ and $\mathrm{B}_{4} ; \mathrm{C}_{1}$, trimer $\mathrm{C}_{1}$; Nd, Not determined. a, b, c; ANOVA was made to compare values obtain between varieties for the same compound. Same letters indicate no significant differences between the value (Tukey's test, $p<0.05$ ). 
Table 2. Total phenol contents, total tannins, total anthocyanins and flavan-3-ol monomers, dimers and trimer characterisation in EAQ and EA70 grape pomace skin extracts.

\begin{tabular}{|c|c|c|c|c|c|c|}
\hline & \multicolumn{6}{|c|}{ Skins-EAQ } \\
\hline & GRE2 a & SYR1 ${ }^{\text {a }}$ & SYR2 ${ }^{\text {a }}$ & CAR $^{\text {a }}$ & MOU $^{a}$ & ALI ${ }^{a}$ \\
\hline TPC & $109.72 \pm 0.19 \mathrm{c}$ & $146.50 \pm 1.19 \mathrm{e}$ & $71.88 \pm 0.08 \mathrm{a}$ & $120.83 \pm 1.12 d$ & $102.27 \pm 0.38 b$ & $196.71 \pm 0.37 f$ \\
\hline Total tannins & $112.28 \pm 2.67 b$ & $156.63 \pm 2.63 \mathrm{c}$ & $86.36 \pm 1.86 \mathrm{a}$ & $161.61 \pm 1.32 \mathrm{c}$ & $104.79 \pm 2.00 \mathrm{~b}$ & $221.4 \pm 3.47 \mathrm{~d}$ \\
\hline Total anthocyanins & $8.70 \pm 0.01 \mathrm{c}$ & $16.01 \pm 0.01 \mathrm{~d}$ & $1.76 \pm 0.01 \mathrm{a}$ & $14.62 \pm 0.75 \mathrm{~d}$ & $5.65 \pm 0.01 \mathrm{~b}$ & $21.40 \pm 0.20 \mathrm{e}$ \\
\hline \multicolumn{7}{|c|}{ Proanthocyanidins composition: } \\
\hline $\mathbf{C}$ & $0.764 \pm 0.003 \mathrm{a}$ & $1.415 \pm 0.012 \mathrm{c}$ & $0.523 \pm 0.057 \mathrm{a}$ & $1.013 \pm 0.003 \mathrm{~b}$ & $0.656 \pm 0.011 \mathrm{a}$ & $2.027 \pm 0.127 \mathrm{~d}$ \\
\hline $\mathbf{E C}$ & $0.285 \pm 0.001 \mathrm{a}$ & $1.043 \pm 0.012 b$ & $0.370 \pm 0.032 \mathrm{a}$ & $0.352 \pm 0.008 \mathrm{a}$ & $0.377 \pm 0.001 \mathrm{a}$ & $1.368 \pm 0.088 \mathrm{c}$ \\
\hline$\Sigma$ Monomers & $1.050 \pm 0.002 \mathrm{ab}$ & $2.460 \pm 0.020 \mathrm{c}$ & $0.890 \pm 0.060 \mathrm{a}$ & $1.360 \pm 0.010 \mathrm{~b}$ & $1.030 \pm 0.010 \mathrm{ab}$ & $3.400 \pm 0.1500 \mathrm{~d}$ \\
\hline $\mathbf{B}_{1}$ & $0.621 \pm 0.002 \mathrm{ab}$ & $0.918 \pm 0.008 b$ & $0.368 \pm 0.003 \mathrm{a}$ & $0.736 \pm 0.007 \mathrm{~b}$ & $0.618 \pm 0.002 \mathrm{ab}$ & $0.908 \pm 0.172 b$ \\
\hline $\mathbf{B}_{2}$ & $0.410 \pm 0.002 \mathrm{a}$ & $0.660 \pm 0.006 \mathrm{bc}$ & $0.363 \pm 0.038 \mathrm{a}$ & $0.433 \pm 0.009 \mathrm{a}$ & $0.568 \pm 0.004 b$ & $0.771 \pm 0.048 \mathrm{c}$ \\
\hline $\mathbf{B}_{3}$ & $0.278 \pm 0.003 \mathrm{a}$ & $0.387 \pm 0.007 \mathrm{a}$ & $0.175 \pm 0.006 \mathrm{a}$ & $0.277 \pm 0.010 \mathrm{a}$ & $0.288 \pm 0.007 \mathrm{a}$ & $0.345 \pm 0.115 \mathrm{a}$ \\
\hline $\mathbf{B}_{4}$ & $\mathrm{Nd}$ & $\mathrm{Nd}$ & $\mathrm{Nd}$ & $\mathrm{Nd}$ & $\mathrm{Nd}$ & $0.317 \pm 0.091$ \\
\hline$\Sigma$ Dimers & $1.310 \pm 0.002 \mathrm{ab}$ & $1.960 \pm 0.004 \mathrm{bc}$ & $0.910 \pm 0.030 \mathrm{a}$ & $1.450 \pm 0.020 \mathrm{ab}$ & $1.470 \pm 0.010 \mathrm{ab}$ & $2.340 \pm 0.300 \mathrm{c}$ \\
\hline \multirow[t]{3}{*}{$\mathbf{C}_{1}$} & $0.469 \pm 0.000 \mathrm{ab}$ & $0.817 \pm 0.012 b$ & $0.344 \pm 0.082 \mathrm{a}$ & $0.537 \pm 0.002 \mathrm{ab}$ & $0.547 \pm 0.125 \mathrm{ab}$ & $0.665 \pm 0.146 \mathrm{ab}$ \\
\hline & \multicolumn{6}{|c|}{ Skins-EA70 } \\
\hline & GRE2 a & SYR1 ${ }^{\text {a }}$ & SYR2 ${ }^{\text {a }}$ & CAR $^{\text {a }}$ & MOU $^{a}$ & ALI ${ }^{a}$ \\
\hline TPC & $195.15 \pm 0.28 \mathrm{c}$ & $224.92 \pm 0.18 \mathrm{f}$ & $173.58 \pm 0.08 \mathrm{a}$ & $203.47 \pm 0.83 \mathrm{~d}$ & $219.88 \pm 0.18 \mathrm{e}$ & $188.94 \pm 0.69 b$ \\
\hline Total tannins & $256.07 \pm 3.65 \mathrm{a}$ & $312.46 \pm 10.77 \mathrm{bc}$ & $250.17 \pm 7.07 \mathrm{a}$ & $345.34 \pm 4.18 \mathrm{c}$ & $268.6 \pm 11.68 \mathrm{ab}$ & $232.65 \pm 3.14 \mathrm{a}$ \\
\hline Total anthocyanins & $53.66 \pm 0.83 a$ & $86.68 \pm 1.71 \mathrm{~b}$ & $45.38 \pm 0.20 \mathrm{a}$ & $88.44 \pm 0.59 b$ & $46.64 \pm 0.39 \mathrm{a}$ & $54.41 \pm 2.66 \mathrm{a}$ \\
\hline \multicolumn{7}{|c|}{ Proanthocyanidins composition: } \\
\hline $\mathbf{C}$ & $1.420 \pm 0.005 \mathrm{a}$ & $2.287 \pm 0.100 \mathrm{~b}$ & $2.094 \pm 0.045 b$ & $1.440 \pm 0.002 \mathrm{a}$ & $1.522 \pm 0.021 \mathrm{a}$ & $5.084 \pm 0.026 \mathrm{c}$ \\
\hline EC & $0.441 \pm 0.003 \mathrm{a}$ & $1.363 \pm 0.008 b$ & $1.101 \pm 0.195 b$ & $0.443 \pm 0.003 \mathrm{a}$ & $0.658 \pm 0.001 \mathrm{a}$ & $2.626 \pm 0.005 \mathrm{c}$ \\
\hline$\Sigma$ Monomers & $1.860 \pm 0.001 \mathrm{a}$ & $3.650 \pm 0.080 \mathrm{c}$ & $3.190 \pm 0.110 b$ & $1.880 \pm 0.003 \mathrm{a}$ & $2.180 \pm 0.020 \mathrm{a}$ & $7.710 \pm 0.020 \mathrm{~d}$ \\
\hline $\mathbf{B}_{1}$ & $0.915 \pm 0.006 \mathrm{a}$ & $1.266 \pm 0.009 b$ & $1.190 \pm 0.164 \mathrm{ab}$ & $1.140 \pm 0.013 \mathrm{ab}$ & $1.150 \pm 0.011 \mathrm{ab}$ & $2.589 \pm 0.004 c$ \\
\hline $\mathbf{B}_{2}$ & $0.396 \pm 0.021 \mathrm{a}$ & $0.635 \pm 0.008 c$ & $0.602 \pm 0.008 b c$ & $0.388 \pm 0.021 \mathrm{a}$ & $0.566 \pm 0.001 b$ & $1.284 \pm 0.001 \mathrm{~d}$ \\
\hline $\mathbf{B}_{3}$ & $0.297 \pm 0.001 b$ & $0.292 \pm 0.003 b$ & $0.360 \pm 0.001 \mathrm{c}$ & $0.265 \pm 0.003 \mathrm{a}$ & $0.305 \pm 0.002 b$ & $0.603 \pm 0.011 d$ \\
\hline $\mathbf{B}_{4}$ & $\mathrm{Nd}$ & $\mathrm{Nd}$ & $\mathrm{Nd}$ & $\mathrm{Nd}$ & $\mathrm{Nd}$ & $0.350 \pm 0.000$ \\
\hline$\Sigma$ Dimers & $1.610 \pm 0.010 \mathrm{a}$ & $2.190 \pm 0.003 c$ & $2.150 \pm 0.110 \mathrm{c}$ & $1.790 \pm 0.030 \mathrm{ab}$ & $2.020 \pm 0.010 \mathrm{bc}$ & $4.830 \pm 0.010 \mathrm{~d}$ \\
\hline $\mathrm{C}_{1}$ & $0.336 \pm 0.002 \mathrm{a}$ & $0.435 \pm 0.001 \mathrm{ab}$ & $0.662 \pm 0.133 b$ & $0.307 \pm 0.002 \mathrm{a}$ & $0.371 \pm 0.004 \mathrm{a}$ & $0.629 \pm 0.003 b$ \\
\hline
\end{tabular}

${ }^{a}$ GRE2, Grenache; SYR1 and SYR2, Syrah; CAR, Carignan; MOU, Mourvèdre, ALI, Alicante. In units of mg GAE/g DW of skins for TPC and mg/g DW of skins for total tannins, total anthocyanins and the quantification of proanthocyanidins. Data are expressed as the mean of triplicate \pm standard deviation. TPC, total phenol contents; C, (+)-Catechin; EC, (-)-Epicatechin; $\mathrm{B}_{1}, \mathrm{~B}_{2} ; \mathrm{B}_{3}, \mathrm{~B}_{4}$, Procyanidin dimers $\mathrm{B}_{1}, \mathrm{~B}_{2} ; \mathrm{B}_{3}, \mathrm{~B}_{4} ; \mathrm{Cl}$, procyanidin trimers $\mathrm{C}_{1} . \Sigma$ Monomers, sum of catechin and epicatechin; $\Sigma$ Dimers, sum of $\mathrm{B}_{1}, \mathrm{~B}_{2}, \mathrm{~B}_{3}$ and $\mathrm{B}_{4} ; \mathrm{C}_{1}$, trimer $\mathrm{C}_{1}$; $\mathrm{Nd}$, Not determined. a, b, c, d, e, f; ANOVA was made to compare values obtain between varieties for the same compound. Same letters indicate no significant differences between the value (Tukey's test, $p<0.05$ ).

Overall, the results showed that the use of a 70\% hydro-alcoholic solution allowed a better extraction of phenolic compounds whether in seeds or in skins. Among the seed extracts, seeds from SYR1 and CAR were particularly rich in polyphenols, tannins and anthocyanins in EAQ and EA70 extracts (Table 1). SYR 1 (EA70) contained a higher tannins concentration, up to $455.42 \mathrm{mg} / \mathrm{g} \mathrm{DW}$, while CAR (EA70) has a higher quantity in total anthocyanins $(57.34 \mathrm{mg} / \mathrm{g} \mathrm{DW})$. In both extracts, phenolic contents in GRE1 were low in comparison with other varieties. Our results are in accordance with several studies which 
have reported a lower amount of total phenol and anthocyanin contents in products derived from Grenache variety compared to other varieties such as Syrah, Mourvèdre and Carignan [24,25]. Indeed, Grenache cultivar is known to be used for rosé or fortified wines production and it is typically blended with other varieties. Moreover Grenache is also known to have thin skin with high ripeness level and high susceptibility to oxidation [26].

For both type of seed extracts, SYR1 was the richest, whether in monomers $(8.88 \mathrm{mg} / \mathrm{g}$ DW in EAQ and $13.84 \mathrm{mg} / \mathrm{g}$ DW in EA70), in dimers $(6.87 \mathrm{mg} / \mathrm{g}$ DW in EAQ and $7.10 \mathrm{mg} / \mathrm{g} \mathrm{DW}$ in EA70) and trimer $\mathrm{C}_{1}(2.00 \mathrm{mg} / \mathrm{g}$ DW in EAQ and $1.25 \mathrm{mg} / \mathrm{g}$ DW in EA70) as opposed to GRE1 (Table 1). The latter had already appeared to contain low amount of polyphenols in previous total analysis. However, despite this low content, GRE1 still possessed an exploitable potential, especially when extracted with $70 \%$ hydro-alcoholic solution.

Concerning skins, results for EAQ extracts revealed that ALI contained the highest phenolic contents for the three tests combined (TPC: $196.71 \mathrm{mg}$ GAE/g DW, total tannins: $221.40 \mathrm{mg} / \mathrm{g}$ DW and total anthocyanins: $21.40 \mathrm{mg} / \mathrm{g}$ DW) while in EA70, SYR1 skins were predominantly high in phenolic contents (total phenol contents: $224.92 \mathrm{mg}$ GAE /g DW, total tannins: $312.46 \mathrm{mg} / \mathrm{g}$ DW and total anthocyanins: $86.68 \mathrm{mg} / \mathrm{g}$ DW) (Table 2). The poorest extract was SYR2, whether in EAQ or in EA70.

In greater detail, EAQ values ranged from $0.89 \mathrm{mg} / \mathrm{g}$ DW to $3.4 \mathrm{mg} / \mathrm{g}$ DW for the sum of monomers, from $0.91 \mathrm{mg} / \mathrm{g}$ DW to $2.34 \mathrm{mg} / \mathrm{g} \mathrm{DW}$ for the sum of dimers and values from $1.88 \mathrm{mg} / \mathrm{g} \mathrm{DW}$ to $7.71 \mathrm{mg} / \mathrm{g} \mathrm{DW}$ and $1.61 \mathrm{mg} / \mathrm{g}$ DW to $4.83 \mathrm{mg} / \mathrm{g} \mathrm{DW}$ were found in EA70 extracts, for the sum of monomers and dimers, respectively. ALI and SYR1 showed a higher content of flavan-3-ol monomers and dimers in both type of extract. Regarding the dimer $\mathrm{B}_{4}$ which could not be found in grape pomace skins except for those of Alicante varieties, a previous study has already reported this phenomenon in grape skins [27,28]. Among EAQ samples, SYR2 skin extracts was evidenced as having fewer amounts than other skin EAQ samples, but in EA70 it was GRE2 and CAR. The difference of polyphenolic content between SYR1 and SYR2 could be explained by the fact that SYR1 and SYR2 were derived from grapes from different parcels. Several studies have demonstrated the importance of climatic and geographical factors and cultural practices [29,30]. Moreover, this difference could also be explained by technical processes $[12,13]$. Actually, SYR1 and SYR2 grapes were used to make different wines and as a result the vinification process employed differed. For instance, in the case of SYR1, fermentation lasted 19 days whereas it lasted 22 day for SYR2.

As it was already observed in seed extracts, EA70 were characterized by higher total phenol contents, total tannins and total anthocyanins. This result illustrated a better extraction by $70 \%$ alcoholic solution. Fournand et al. [31] reported that tannin extraction efficiency in a hydroalcoholic solution similar to wine was lower than $38 \%$. Indeed only a small amount of tannins are released during fermentation and this resulted in a fermented pomace with high tannin contents and increased tannin extractibility. Ethanol can facilitate tissue dissolution and thus, liberate a greater amount of polyphenols. Actually, between EAQ and EA70, total polyphenols and total tannins rate were 1.5- to 2.5- and 1- to 3-fold respectively, higher in EA70. Furthermore, beside the solvent effect, previous studies by Vergara-Salinas et al. [32] have shown that fermented pomace could also facilitate the extraction of tannins compared to unfermented ones. 


\subsection{Anthocyanin Analysis of Grape Pomace Seed and Skin Extracts by HPLC-UV-MS}

In total, 18 anthocyanins were detected by HPLC-PDA-MS. Compounds were identified on the basis of their absorbance spectra, the retention times of commercially available standards, elution order, $\mathrm{m} / \mathrm{z}$ of the positively charged molecular ion $\left([\mathrm{M}]^{+}\right)$and on the $\mathrm{MS}^{2}$ fragmentation, according to previous reports. Tables 3 and 4 summarize the anthocyanin contents of grape pomace seed and skin extracts in which total anthocyanins represents the sum of individual anthocyanins.

Inall studied varieties, the main compounds consisted of 3-O-monoglucosides $\left([\mathrm{M}-162]^{+}\right)$of delphinidin ( $\mathrm{m} / \mathrm{z} 303)$, cyanidin $(\mathrm{m} / \mathrm{z} 287)$, petunidin $(\mathrm{m} / \mathrm{z} 317)$, peonidin $(\mathrm{m} / \mathrm{z} 301)$ and malvidin $(\mathrm{m} / \mathrm{z} 331)$ which accounted for 63\% in EAQ, 64\% in EA70 to 72\% in EAQ, 70\% in EA70 of the total anthocyanins content in seed and skin pomace extracts, respectively.

Table 3. Anthocyanin characterisation in EAQ and EA70 grape pomace seed extracts.

\begin{tabular}{|c|c|c|c|c|c|c|}
\hline & \multicolumn{3}{|c|}{ Seeds-EAQ } & \multicolumn{3}{|c|}{ Seeds-EA70 } \\
\hline & GRE1 $^{\text {a }}$ & SYR1 a $^{\text {a }}$ & $\mathrm{CAR}^{\mathrm{a}}$ & GRE1 $^{a}$ & SYR1 a & $\mathrm{CAR}^{\mathrm{a}}$ \\
\hline Dp-3-O-Glc & $0.03 \pm 0.00 \mathrm{a}$ & $0.08 \pm 0.00 \mathrm{~b}$ & $0.40 \pm 0.00 \mathrm{c}$ & $0.19 \pm 0.00 \mathrm{a}$ & $0.31 \pm 0.00 \mathrm{~b}$ & $3.11 \pm 0.02 \mathrm{c}$ \\
\hline Cy-3-O-Glc & $0.02 \pm 0.00 \mathrm{a}$ & $0.02 \pm 0.00 \mathrm{a}$ & $0.05 \pm 0.00 \mathrm{~b}$ & $0.09 \pm 0.00 \mathrm{~b}$ & $0.05 \pm 0.00 \mathrm{a}$ & $0.23 \pm 0.00 \mathrm{c}$ \\
\hline Pt-3-O-Glc & $0.05 \pm 0.00 \mathrm{a}$ & $0.14 \pm 0.00 \mathrm{~b}$ & $0.43 \pm 0.00 \mathrm{c}$ & $0.33 \pm 0.01 \mathrm{a}$ & $0.58 \pm 0.02 b$ & $3.18 \pm 0.01 \mathrm{c}$ \\
\hline Pn-3-O-Glc & $0.11 \pm 0.00 \mathrm{a}$ & $0.12 \pm 0.00 \mathrm{~b}$ & $0.15 \pm 0.01 \mathrm{c}$ & $0.56 \pm 0.03 \mathrm{a}$ & $0.47 \pm 0.02 \mathrm{a}$ & $1.11 \pm 0.00 \mathrm{~b}$ \\
\hline Mv-3-O-Glc & $0.39 \pm 0.00 \mathrm{a}$ & $0.93 \pm 0.00 \mathrm{~b}$ & $1.48 \pm 0.02 \mathrm{c}$ & $2.36 \pm 0.12 \mathrm{a}$ & $3.57 \pm 0.08 b$ & $10.52 \pm 0.11 \mathrm{c}$ \\
\hline Mv-3-O-Glc-acetaldehyde (vitisin B) & $\mathrm{Nd}$ & $0.02 \pm 0.00 \mathrm{~b}$ & $0.02 \pm 0.00 \mathrm{a}$ & $0.01 \pm 0.00 \mathrm{a}$ & $0.05 \pm 0.00 \mathrm{c}$ & $0.03 \pm 0.00 \mathrm{~b}$ \\
\hline Dp-3-O-(6"-O-acetyl)-Glc & $\mathrm{Nd}$ & $0.02 \pm 0.00$ & $\mathrm{Nd}$ & $\mathrm{Nd}$ & $0.09 \pm 0.00 \mathrm{~b}$ & $0.06 \pm 0.00 \mathrm{a}$ \\
\hline Dimer Mv-Cat & $0.01 \pm 0.00 \mathrm{a}$ & $0.03 \pm 0.00 \mathrm{~b}$ & $\mathrm{Nd}$ & $0.04 \pm 0.00 \mathrm{a}$ & $0.05 \pm 0.00 \mathrm{~b}$ & $0.06 \pm 0.00 \mathrm{c}$ \\
\hline Mv-3-O-glc-pyruvate (vitisin A) & $0.02 \pm 0.00 \mathrm{a}$ & $0.04 \pm 0.00 \mathrm{~b}$ & $0.04 \pm 0.00 \mathrm{~b}$ & $0.12 \pm 0.00 \mathrm{~b}$ & $0.07 \pm 0.00 \mathrm{a}$ & $0.14 \pm 0.00 \mathrm{c}$ \\
\hline Dimer Mv-Cat & $0.002 \pm 0.00 \mathrm{a}$ & $0.07 \pm 0.00 \mathrm{c}$ & $0.05 \pm 0.00 \mathrm{~b}$ & $\mathrm{Nd}$ & $0.13 \pm 0.00 \mathrm{a}$ & $0.17 \pm 0.00 \mathrm{~b}$ \\
\hline Dimer Mv-Cat & $0.01 \pm 0.00 \mathrm{a}$ & $0.03 \pm 0.00 \mathrm{c}$ & $0.02 \pm 0.00 \mathrm{~b}$ & $0.10 \pm 0.00 \mathrm{a}$ & $0.13 \pm 0.00 \mathrm{~b}$ & $0.16 \pm 0.00 \mathrm{c}$ \\
\hline Pn-3-O-(6"-O-acetyl)-Glc & $0.01 \pm 0.00 \mathrm{a}$ & $0.07 \pm 0.00 \mathrm{~b}$ & $\mathrm{Nd}$ & $0.07 \pm 0.00 \mathrm{a}$ & $0.32 \pm 0.01 \mathrm{c}$ & $0.12 \pm 0.00 \mathrm{~b}$ \\
\hline Mv-3-O-(6"-O-acetyl)-Glc & $0.02 \pm 0.00 \mathrm{a}$ & $0.32 \pm 0.00 \mathrm{c}$ & $0.09 \pm 0.00 \mathrm{~b}$ & $0.06 \pm 0.00 \mathrm{a}$ & $0.9 \pm 0.03 \mathrm{c}$ & $0.31 \pm 0.00 \mathrm{~b}$ \\
\hline Dp-3-O-(6"-O-coumaroyl)-Glc & $0.005 \pm 0.00 \mathrm{a}$ & $0.06 \pm 0.00 \mathrm{~b}$ & $0.10 \pm 0.00 \mathrm{c}$ & $0.05 \pm 0.00 \mathrm{a}$ & $0.18 \pm 0.00 \mathrm{~b}$ & $0.58 \pm 0.01 \mathrm{c}$ \\
\hline Mv-3-O-(6"-O-caffeoyl)-Glc & $0.01 \pm 0.00 \mathrm{a}$ & $0.04 \pm 0.00 \mathrm{c}$ & $0.02 \pm 0.00 \mathrm{~b}$ & $0.08 \pm 0.00 \mathrm{a}$ & $0.11 \pm 0.00 \mathrm{~b}$ & $0.33 \pm 0.00 \mathrm{c}$ \\
\hline Cy-3-O-(6"-O-coumaroyl)-Glc & $0.01 \pm 0.00 \mathrm{a}$ & $0.02 \pm 0.00 \mathrm{~b}$ & $0.02 \pm 0.00 \mathrm{~b}$ & $0.07 \pm 0.00 \mathrm{a}$ & $0.07 \pm 0.00 \mathrm{a}$ & $0.11 \pm 0.00 \mathrm{~b}$ \\
\hline Pt-3-O-(6"-O-coumaroyl)-Glc & $0.01 \pm 0.00 \mathrm{a}$ & $0.11 \pm 0.00 \mathrm{~b}$ & $0.11 \pm 0.00 \mathrm{~b}$ & $0.08 \pm 0.00 \mathrm{a}$ & $0.35 \pm 0.01 \mathrm{~b}$ & $0.66 \pm 0.00 \mathrm{c}$ \\
\hline Mv-3-O-(6"-O-coumaroyl)-Glc & $0.06 \pm 0.00 \mathrm{a}$ & $1.14 \pm 0.01 \mathrm{c}$ & $0.65 \pm 0.00 \mathrm{~b}$ & $0.80 \pm 0.00 \mathrm{a}$ & $2.85 \pm 0.00 \mathrm{~b}$ & $4.51 \pm 0.04 \mathrm{c}$ \\
\hline Total anthocyanins Glc & $0.60 \pm 0.00 \mathrm{a}$ & $1.30 \pm 0.00 b$ & $2.52 \pm 0.03 \mathrm{c}$ & $3.53 \pm 0.16 \mathrm{a}$ & $4.99 \pm 0.12 b$ & $18.15 \pm 0.09 \mathrm{c}$ \\
\hline Total anthocyanins acetylated & $0.03 \pm 0.00 \mathrm{a}$ & $0.41 \pm 0.00 \mathrm{c}$ & $0.09 \pm 0.00 b$ & $0.13 \pm 0.00 \mathrm{a}$ & $1.30 \pm 0.02 c$ & $0.49 \pm 0.00 b$ \\
\hline Total anthocyanins coumaroylated & $0.08 \pm 0.00 \mathrm{a}$ & $1.32 \pm 0.01 \mathrm{c}$ & $0.88 \pm 0.00 b$ & $1.00 \pm 0.00 \mathrm{a}$ & $3.44 \pm 0.01 b$ & $5.86 \pm 0.02 c$ \\
\hline Total anthocyanins & $0.76 \pm 0.00 \mathrm{a}$ & $3.26 \pm 0.01 b$ & $3.63 \pm 0.03 c$ & $5.00 \pm 0.17 \mathrm{a}$ & $10.28 \pm 0.14 b$ & $25.38 \pm 0.11 \mathrm{c}$ \\
\hline
\end{tabular}

${ }^{a}$ GRE1, Grenache; SYR1, Syrah; CAR, Carignan; Dp, Delphinidin; Cy, Cyanidin; Pt, Petunidin; Pn, Peonidin, Mv, Malvidin; Cat, Catechin; Glc, glucoside; Nd, not determined. Data are expressed as the mean of triplicate \pm standard deviation as mg malvidin-3-O-glucoside equivalents/g DW of skins. a, b, c; ANOVA was made to compare values obtain between varieties for the same compound. Same letters indicate no significant differences between the value (Tukey's test, $p<0.05$ ). 
Table 4. Anthocyanin characterisation in EAQ and EA70 grape pomace skin extracts.

\begin{tabular}{|c|c|c|c|c|c|c|}
\hline & \multicolumn{6}{|c|}{ Skins-EAQ } \\
\hline & GRE2 a & SYR1 $^{\text {a }}$ & SYR2 a & CAR $^{a}$ & MOU $^{a}$ & $\mathbf{A L I}^{\text {a }}$ \\
\hline Dp-3-O-glc & $0.24 \pm 0.01 \mathrm{~d}$ & $0.15 \pm 0.00 \mathrm{~b}$ & $0.01 \pm 0.00 \mathrm{a}$ & $0.53 \pm 0.00 \mathrm{f}$ & $0.20 \pm 0.00 \mathrm{c}$ & $0.31 \pm 0.00 \mathrm{e}$ \\
\hline Cy-3-O-glc & $0.09 \pm 0.00 \mathrm{~d}$ & $0.01 \pm 0.00 \mathrm{a}$ & $\mathrm{Nd}$ & $0.05 \pm 0.00 \mathrm{~b}$ & $0.07 \pm 0.00 \mathrm{c}$ & $0.11 \pm 0.00 \mathrm{e}$ \\
\hline Pt-3-O-glc & $0.33 \pm 0.01 \mathrm{c}$ & $0.25 \pm 0.01 \mathrm{~b}$ & $0.03 \pm 0.00 \mathrm{a}$ & $0.54 \pm 0.00 \mathrm{e}$ & $0.30 \pm 0.00 \mathrm{c}$ & $0.47 \pm 0.02 \mathrm{~d}$ \\
\hline Pn-3-O-glc & $0.35 \pm 0.01 \mathrm{c}$ & $0.2 \pm 0.01 \mathrm{~b}$ & $0.02 \pm 0.00 \mathrm{a}$ & $0.19 \pm 0.00 \mathrm{~b}$ & $0.22 \pm 0.00 \mathrm{~b}$ & $1.49 \pm 0.03 \mathrm{~d}$ \\
\hline Mv-3-O-glc & $1.78 \pm 0.05 \mathrm{~d}$ & $1.54 \pm 0.05 \mathrm{c}$ & $0.11 \pm 0.00 \mathrm{a}$ & $1.74 \pm 0.02 \mathrm{~d}$ & $1.01 \pm 0.01 \mathrm{~b}$ & $3.14 \pm 0.03 \mathrm{e}$ \\
\hline Mv-3-O-glc-acetaldehyde (vitisin B) & $0.01 \pm 0.00 \mathrm{a}$ & $0.02 \pm 0.00 \mathrm{~b}$ & $\mathrm{Nd}$ & $0.02 \pm 0.00 \mathrm{~b}$ & $0.01 \pm 0.00 \mathrm{a}$ & $\mathrm{Nd}$ \\
\hline Dp-3-O-(6"-O-acetyl) glc & $\mathrm{Nd}$ & $0.05 \pm 0.00 \mathrm{~b}$ & $\mathrm{Nd}$ & $0.02 \pm 0.00 \mathrm{a}$ & $\mathrm{Nd}$ & $\mathrm{Nd}$ \\
\hline Dimer Mv-Cat & $0.01 \pm 0.00 \mathrm{a}$ & $0.03 \pm 0.00 \mathrm{~b}$ & $\mathrm{Nd}$ & $\mathrm{Nd}$ & $0.01 \pm 0.00 \mathrm{a}$ & $0.03 \pm 0.00 \mathrm{~b}$ \\
\hline Mv-3-O-glc-pyruvate (vitisin A) & $0.05 \pm 0.00 \mathrm{c}$ & $0.04 \pm 0.00 \mathrm{bc}$ & $0.02 \pm 0.00 \mathrm{a}$ & $0.04 \pm 0.00 \mathrm{~b}$ & $0.06 \pm 0.00 \mathrm{~d}$ & $0.10 \pm 0.00 \mathrm{e}$ \\
\hline Dimer Mv-Cat & $\mathrm{Nd}$ & $0.08 \pm 0.00 \mathrm{c}$ & $\mathrm{Nd}$ & $0.05 \pm 0.00 \mathrm{~b}$ & $0.01 \pm 0.00 \mathrm{a}$ & $0.05 \pm 0.00 \mathrm{~b}$ \\
\hline Dimer Mv-Cat & $0.01 \pm 0.00 \mathrm{bc}$ & $0.03 \pm 0.00 \mathrm{~d}$ & $0.01 \pm 0.00 \mathrm{c}$ & $0.01 \pm 0.00 \mathrm{a}$ & $0.01 \pm 0.00 \mathrm{ab}$ & $0.03 \pm 0.00 \mathrm{~d}$ \\
\hline Pn-3-O-(6"-O-acetyl)-glc & $0.02 \pm 0.00 \mathrm{c}$ & $0.10 \pm 0.00 \mathrm{e}$ & $0.01 \pm 0.00 \mathrm{a}$ & $0.02 \pm 0.00 \mathrm{~b}$ & $0.01 \pm 0.00 \mathrm{ab}$ & $0.09 \pm 0.00 \mathrm{~d}$ \\
\hline Mv-3-O-(6"-O-acetyl)-glc & $\mathrm{Nd}$ & $0.52 \pm 0.00 \mathrm{e}$ & $0.02 \pm 0.00 \mathrm{a}$ & $0.07 \pm 0.00 \mathrm{c}$ & $0.04 \pm 0.00 \mathrm{~b}$ & $0.19 \pm 0.00 \mathrm{~d}$ \\
\hline Dp-3-O-(6"-O-coumaroyl)-glc & $\mathrm{Nd}$ & $0.09 \pm 0.00 \mathrm{c}$ & $\mathrm{Nd}$ & $0.13 \pm 0.00 \mathrm{~d}$ & $0.02 \pm 0.00 \mathrm{a}$ & $0.06 \pm 0.00 \mathrm{~b}$ \\
\hline Mv-3-O-(6"-O-caffeoyl)-glc & $0.02 \pm 0.00 \mathrm{a}$ & $0.05 \pm 0.00 \mathrm{c}$ & $\mathrm{Nd}$ & $0.03 \pm 0.00 \mathrm{~b}$ & $0.02 \pm 0.00 \mathrm{a}$ & $0.05 \pm 0.00 \mathrm{c}$ \\
\hline Сy-3-O-(6"-O-coumaroyl)-glc & $0.01 \pm 0.00 \mathrm{a}$ & $0.02 \pm 0.00 \mathrm{~b}$ & $\mathrm{Nd}$ & $0.03 \pm 0.00 \mathrm{c}$ & $0.03 \pm 0.00 \mathrm{~d}$ & $0.03 \pm 0.00 \mathrm{c}$ \\
\hline Pt-3-O-(6"-O-coumaroyl)-glc & $0.04 \pm 0.00 \mathrm{~b}$ & $0.16 \pm 0.00 \mathrm{e}$ & $\mathrm{Nd}$ & $0.12 \pm 0.00 \mathrm{~d}$ & $0.03 \pm 0.00 \mathrm{a}$ & $0.07 \pm 0.00 \mathrm{c}$ \\
\hline Mv-3-O-(6"-O-coumaroyl)-glc & $0.22 \pm 0.00 \mathrm{c}$ & $1.59 \pm 0.00 \mathrm{f}$ & $0.01 \pm 0.00 \mathrm{a}$ & $0.68 \pm 0.01 \mathrm{~d}$ & $0.15 \pm 0.00 \mathrm{~b}$ & $1.11 \pm 0.01 \mathrm{e}$ \\
\hline Total anthocyanins glc & $2.8 \pm 0.07 \mathrm{~d}$ & $2.15 \pm 0.06 c$ & $0.17 \pm 0.00 \mathrm{a}$ & $3.05 \pm 0.02 \mathrm{e}$ & $1.8 \pm 0.01 b$ & $5.52 \pm 0.08 f$ \\
\hline Total anthocyanins acetylated & $0.02 \pm 0.00 \mathrm{a}$ & $0.67 \pm 0.00 \mathrm{e}$ & $0.03 \pm 0.00 \mathrm{a}$ & $0.11 \pm 0.00 c$ & $0.05 \pm 0.00 b$ & $0.27 \pm 0.00 \mathrm{~d}$ \\
\hline Total anthocyanins coumaroylated & $0.27 \pm 0.00 \mathrm{c}$ & $1.86 \pm 0.00 f$ & $0.01 \pm 0.00 \mathrm{a}$ & $0.96 \pm 0.01 \mathrm{~d}$ & $0.24 \pm 0.00 b$ & $1.27 \pm 0.01 \mathrm{e}$ \\
\hline \multirow[t]{3}{*}{ Total anthocyanins } & $3.19 \pm 0.08 \mathrm{c}$ & $4.92 \pm 0.06 \mathrm{e}$ & $0.24 \pm 0.00 \mathrm{a}$ & $4.25 \pm 0.03 \mathrm{~d}$ & $2.2 \pm 0.01 b$ & $7.32 \pm \mathbf{0 . 0 8 f}$ \\
\hline & \multicolumn{6}{|c|}{ Skins-EA70 } \\
\hline & GRE2 $^{\text {a }}$ & SYR1 a & SYR2 $^{\text {a }}$ & $\mathrm{CAR}^{\mathrm{a}}$ & MOU $^{a}$ & $\mathbf{A L I}^{\mathbf{a}}$ \\
\hline Dp-3-O-glc & $1.43 \pm 0.00 \mathrm{~b}$ & $0.78 \pm 0.00 \mathrm{a}$ & $0.97 \pm 0.00 \mathrm{a}$ & $5.35 \pm 0.21 \mathrm{~d}$ & $2.35 \pm 0.00 \mathrm{c}$ & $1.06 \pm 0.01 \mathrm{a}$ \\
\hline Су-3-O-glc & $0.34 \pm 0.02 \mathrm{~d}$ & $0.05 \pm 0.00 \mathrm{a}$ & $0.12 \pm 0.00 \mathrm{~b}$ & $0.39 \pm 0.01 \mathrm{e}$ & $0.52 \pm 0.00 \mathrm{f}$ & $0.24 \pm 0.00 \mathrm{c}$ \\
\hline Pt-3-O-glc & $2.05 \pm 0.08 \mathrm{~b}$ & $1.29 \pm 0.00 \mathrm{a}$ & $1.53 \pm 0.00 \mathrm{a}$ & $5.04 \pm 0.21 \mathrm{~d}$ & $3.38 \pm 0.02 \mathrm{c}$ & $1.65 \pm 0.03 \mathrm{a}$ \\
\hline Pn-3-O-glc & $1.91 \pm 0.02 \mathrm{c}$ & $0.87 \pm 0.01 \mathrm{a}$ & $0.94 \pm 0.02 \mathrm{a}$ & $1.71 \pm 0.03 \mathrm{~b}$ & $2.00 \pm 0.01 \mathrm{~d}$ & $5.32 \pm 0.01 \mathrm{e}$ \\
\hline Mv-3-O-glc & $10.96 \pm 0.22 \mathrm{c}$ & $7.59 \pm 0.04 b$ & $6.76 \pm 0.09 \mathrm{a}$ & $14.82 \pm 0.42 \mathrm{~d}$ & $10.55 \pm 0.03 \mathrm{c}$ & $11.18 \pm 0.05 \mathrm{c}$ \\
\hline Mv-3-O-glc-acetaldehyde (vitisin B) & $0.03 \pm 0.00 \mathrm{~b}$ & $0.06 \pm 0.00 \mathrm{e}$ & $0.07 \pm 0.00 \mathrm{f}$ & $0.05 \pm 0.00 \mathrm{~d}$ & $0.04 \pm 0.00 \mathrm{c}$ & $0.03 \pm 0.00 \mathrm{a}$ \\
\hline Dp-3-O-(6"-O-acetyl) glc & $0.04 \pm 0.00 \mathrm{a}$ & $0.16 \pm 0.00 \mathrm{e}$ & $0.13 \pm 0.00 \mathrm{~d}$ & $0.09 \pm 0.00 \mathrm{c}$ & $0.05 \pm 0.00 \mathrm{ab}$ & $0.05 \pm 0.00 \mathrm{~b}$ \\
\hline Dimer Mv-Cat & $0.05 \pm 0.00 \mathrm{a}$ & $0.07 \pm 0.00 \mathrm{c}$ & $0.11 \pm 0.00 \mathrm{e}$ & $\mathrm{Nd}$ & $0.10 \pm 0.00 \mathrm{~d}$ & $0.06 \pm 0.00 \mathrm{~b}$ \\
\hline Mv-3-O-glc-pyruvate (vitisin A) & $0.22 \pm 0.01 \mathrm{c}$ & $0.17 \pm 0.01 \mathrm{a}$ & $0.39 \pm 0.00 \mathrm{e}$ & $0.18 \pm 0.01 \mathrm{a}$ & $0.32 \pm 0.00 \mathrm{~d}$ & $0.20 \pm 0.00 \mathrm{~b}$ \\
\hline Dimer Mv-Cat & $0.12 \pm 0.00 \mathrm{~b}$ & $0.27 \pm 0.01 \mathrm{e}$ & $0.21 \pm 0.00 \mathrm{~d}$ & $0.16 \pm 0.00 \mathrm{c}$ & $0.07 \pm 0.00 \mathrm{a}$ & $0.14 \pm 0.00 \mathrm{~b}$ \\
\hline Dimer Mv-Cat & $0.10 \pm 0.00 \mathrm{~b}$ & $0.11 \pm 0.00 \mathrm{c}$ & $0.21 \pm 0.01 \mathrm{~d}$ & $0.10 \pm 0.00 \mathrm{~b}$ & $0.08 \pm 0.00 \mathrm{a}$ & $0.23 \pm 0.00 \mathrm{e}$ \\
\hline Pn-3-O-(6"-O-acetyl)-glc & $0.17 \pm 0.00 \mathrm{a}$ & $0.52 \pm 0.01 \mathrm{c}$ & $0.39 \pm 0.01 \mathrm{bc}$ & $0.10 \pm 0.00 \mathrm{a}$ & $0.19 \pm 0.00 \mathrm{ab}$ & $0.52 \pm 0.14 \mathrm{c}$ \\
\hline Mv-3-O-(6"-O-acetyl)-glc & $0.46 \pm 0.01 \mathrm{a}$ & $2.11 \pm 0.05 \mathrm{~d}$ & $1.06 \pm 0.00 \mathrm{c}$ & $0.41 \pm 0.02 \mathrm{a}$ & $0.39 \pm 0.00 \mathrm{a}$ & $0.61 \pm 0.00 \mathrm{~b}$ \\
\hline Dp-3-O-(6"-O-coumaroyl)-glc & $0.25 \pm 0.01 \mathrm{~b}$ & $0.12 \pm 0.00 \mathrm{a}$ & $0.35 \pm 0.01 \mathrm{e}$ & $0.75 \pm 0.00 \mathrm{f}$ & $0.30 \pm 0.00 \mathrm{~d}$ & $0.27 \pm 0.00 \mathrm{c}$ \\
\hline Mv-3-O-(6"-O-caffeoyl)-glc & $0.34 \pm 0.01 \mathrm{~d}$ & $0.15 \pm 0.00 \mathrm{a}$ & $0.56 \pm 0.00 \mathrm{e}$ & $0.24 \pm 0.00 \mathrm{~b}$ & $0.32 \pm 0.00 \mathrm{c}$ & $0.25 \pm 0.00 \mathrm{~b}$ \\
\hline Cy-3-O-(6"-O-coumaroyl)-glc & $0.13 \pm 0.00 \mathrm{~b}$ & $0.05 \pm 0.00 \mathrm{a}$ & $0.17 \pm 0.01 \mathrm{~d}$ & $0.13 \pm 0.00 \mathrm{~b}$ & $0.52 \pm 0.00 \mathrm{e}$ & $0.14 \pm 0.00 \mathrm{c}$ \\
\hline Pt-3-O-(6"-O-coumaroyl)-glc & $0.36 \pm 0.00 \mathrm{~b}$ & $0.20 \pm 0.00 \mathrm{a}$ & $0.51 \pm 0.01 \mathrm{c}$ & $0.82 \pm 0.01 \mathrm{f}$ & $0.65 \pm 0.00 \mathrm{e}$ & $0.55 \pm 0.00 \mathrm{~d}$ \\
\hline Mv-3-O-(6"-O-coumaroyl)-glc & $3.09 \pm 0.02 b$ & $1.53 \pm 0.00 \mathrm{a}$ & $4.12 \pm 0.00 \mathrm{e}$ & $3.80 \pm 0.03 \mathrm{~d}$ & $3.24 \pm 0.00 \mathrm{c}$ & $6.25 \pm 0.04 f$ \\
\hline Total anthocyanins glc & $16.68 \pm 0.34 b$ & $10.59 \pm 0.05 a$ & $10.33 \pm 0.10 \mathrm{a}$ & $27.3 \pm 0.88 d$ & $18.79 \pm 0.02 \mathrm{c}$ & $19.45 \pm 0.08 \mathrm{c}$ \\
\hline
\end{tabular}


Table 4. Cont.

\begin{tabular}{|c|c|c|c|c|c|c|}
\hline & \multicolumn{6}{|c|}{ Skins-EA 70} \\
\hline & GRE2 a & SYR1 a & SYR2 a & CAR a & MOU a & ALI ${ }^{a}$ \\
\hline Total anthocyanins acetylated & $0.67 \pm 0.01 a$ & $2.79 \pm 0.04 d$ & $1.59 \pm 0.01 \mathrm{c}$ & $0.60 \pm 0.02 \mathrm{a}$ & $0.62 \pm 0.00 \mathrm{a}$ & $1.18 \pm 0.14 b$ \\
\hline Total anthocyanins coumaroylated & $3.82 \pm 0.01 b$ & $1.89 \pm 0.00 \mathrm{a}$ & $5.14 \pm 0.01 d$ & $5.494 \pm 0.04 \mathrm{e}$ & $4.71 \pm 0.00 \mathrm{c}$ & $7.21 \pm 0.04 f$ \\
\hline Total anthocyanins & $22.03 \pm 0.35 c$ & $16.10 \pm 0.10 \mathrm{a}$ & $18.60 \pm 0.11 b$ & $34.11 \pm 0.95 f$ & $25.06 \pm 0.01 d$ & $28.74 \pm 0.01 \mathrm{e}$ \\
\hline
\end{tabular}

a GRE2. Grenache; SYR1 and SYR2. Syrah; CAR. Carignan; MOU. Mourvèdre; ALI. Alicante; Dp, Delphinidin; Cy, Cyanidin; Pt, Petunidin; Pn, Peonidin, Mv, Malvidin, Cat, Catechin; Glc, glucoside; Nd. not determined. Data are expressed as the mean of triplicate \pm standard deviation as mg malvidin-3-O-glucoside equivalents/g DW of skins. a, b, c, d, e, f; ANOVA was made to compare values obtain between varieties for the same compound. Same letters indicate no significant differences between the value (Tukey's test, $p<0.05$ ).

Other compounds were largely represented by 3-O-(6"-O-coumaroyl) glucoside anthocyanins $\left([\mathrm{M}-308]^{+}\right)$followed by the 3-O-(6"-O-acetyl) glucoside one $\left([\mathrm{M}-204]^{+}\right)$(Tables 3 and 4$)$. These results are in agreement with previously reported data which illustrated the predominantly monoglucoside character of $V$. vinifera species [33-35]. Besides, malvidin-3-O-glucoside and its derivatives, $p$-coumaroyl derivatives, petunidin-3-O-glucoside and peonidin-3-O-glucoside were the major compounds. Malvidin-3-O-glucoside alone accounted for $30 \%$ in seeds to $40 \%$ in skins, whereas the minor compound cyanidin-3-O-glucoside represented no more than $2 \%$ of the total anthocyanins.

In seed pomace extracts, an appreciable amount of anthocyanins still remained. This is due, in the first instance, to the contact between seeds and skins throughout the winemaking process, in particular during the pressing and maceration. Moreover, despite the separation of skins from seeds, the operation was not complete and some skins residues remained.

In the aqueous extracts, total anthocyanin contents ranged from $0.76 \pm 0.001 \mathrm{mg} / \mathrm{g} \mathrm{DW}$ to $3.63 \pm 0.03 \mathrm{mg} / \mathrm{g} \mathrm{DW}$ in GRE1 and CAR, respectively. CAR samples possessed the highest level of 3-O-glucoside anthocyanins $(2.52 \pm 0.03 \mathrm{mg} / \mathrm{g} \mathrm{DW})$ while SYR1 contained more acetylated and coumaroylated anthocyanins $(0.41 \pm 0.001 \mathrm{mg} / \mathrm{g}$ DW and $1.32 \pm 0.01 \mathrm{mg} / \mathrm{g} \mathrm{DW}$ respectively). Extracts of these two varieties contained four times more anthocyanins than Grenache, which was composed of only $0.76 \pm 0.001 \mathrm{mg} / \mathrm{g}$ DW of total anthocyanins (Table 3 ).

Regarding the $70 \%$ hydro-alcoholic extract, overall, the level of anthocyanins was higher: 6.6-, 3.2and 7-fold more anthocyanins were extracted from GRE1, SYR1 and CAR, respectively, than with the aqueous extraction method. Total anthocyanin levels ranged from $5.00 \pm 0.17 \mathrm{mg} / \mathrm{g} \mathrm{DW}$ in GRE1 to $25.38 \pm 0.11 \mathrm{mg} / \mathrm{g} \mathrm{DW}$ in CAR, which also possessed the highest levels of 3-O-glucosides and 3-O-(6"-O-coumaroyl) glucoside anthocyanins $(18.15 \pm 0.09 \mathrm{mg} / \mathrm{g} \mathrm{DW}$ and $5.86 \pm 0.02 \mathrm{mg} / \mathrm{g} \mathrm{DW}$ respectively). Besides, an important amount of 3-O-(6"-O-acetyl) glucoside $(1.3 \pm 0.02 \mathrm{mg} / \mathrm{g} \mathrm{DW})$ was founded in SYR1 grape pomace seeds. Among the three studied varieties, the CAR variety proved to be a promising source of anthocyanins, especially in the EA70 extract compared to EAQ and the two other varieties. Data concerning Grenache are in good agreement with those obtained in total analysis (Table 1) and other comparative studies [24,25].

As expected, anthocyanin levels in skin pomace extracts were higher than those in seeds and the predominant compound was malvidin-3-O-glucoside, followed by petudinin-3-O-glucoside and peonidin-3-O-glucoside (Table 4). Previous studies showed that anthocyanins are extracted mainly in the aqueous phase during maceration prior to fermentation and at the beginning of alcoholic fermentation. 
Even though up to $77 \%$ of anthocyanins could be released in this process [31], a surprisingly large amount of anthocyanins still remained in grape pomace skins after the vinification process.

In aqueous extracts, the total anthocyanins value ranged from $0.24 \pm 0.001 \mathrm{mg} / \mathrm{g} \mathrm{DW}$ in SYR2 to $7.32 \pm 0.08 \mathrm{mg} / \mathrm{g}$ DW in ALI. SYR1 possessed appreciable levels especially 3-O-(6"-O-acetyl) glucoside and 3-O-(6"-O-coumaroyl) glucoside anthocyanins. Concerning the $70 \%$ hydro-alcoholic extracts, levels ranged from $16.1 \pm 0.1 \mathrm{mg} / \mathrm{g} \mathrm{DW}$ in SYR1 to $34.11 \pm 0.95 \mathrm{mg} / \mathrm{g} \mathrm{DW}$ in CAR. Moreover, $\mathrm{CAR}$, ALI and MOU were the varieties which possessed the highest amounts of anthocyanin 3-O-glucosides. Regarding the 3-O-(6"-O-acetyl) glucoside anthocyanins, the two Syrah (SYR1 and SYR2) retained the highest amounts reaching $2.79 \pm 0.04 \mathrm{mg} / \mathrm{g}$ DW and $1.59 \pm 0.01 \mathrm{mg} / \mathrm{g} \mathrm{DW}$, respectively. The 3-O-(6"-O-coumaroyl) glucoside anthocyanins were predominant in ALI, CAR and SYR2 (Table 4).

Considering the difference between the two extraction methods, the extraction yield was superior in $70 \%$ hydro-alcoholic extracts with the amounts, depending on the variety, varying from 3- to 77-fold higher. Surprisingly, in SYR2, 77 times more total anthocyanins were extracted. The level of 3-O-(6"-O-acetyl) glucoside and 3-O-(6"-O-coumaroyl) glucoside anthocyanins reached up to $1.59 \pm 0.01 \mathrm{mg} / \mathrm{g}$ DW and $5.14 \pm 0.01 \mathrm{mg} / \mathrm{g}$ DW, respectively. However, the two different methods of extraction did not strictly increase or decrease the ratio of 3-O-glucoside, 3-O-(6"-O-acetyl) glucoside and 3-O-(6"-O-coumaroyl) glucoside in grape skin and seed pomace extracts (Tables 3 and 4). In some varieties, using $70 \%$ hydro-alcoholic extraction increased the relative amount of 3-O-(6"-O-coumaroyl) glucoside obtained, but in other instances, it decreased the ratio. For instance, GRE1 skin pomace extract comprised 9\% coumaroylated anthocyanins in EAQ which increased to $17 \%$ when using $70 \%$ alcohol whereas in SYR1, 38\% were extracted in EAQ and the ratio decreased to $12 \%$ in EA70. Overall, the data showed that the two different extraction methods did not substantially affect the ratio of 3-O-glucoside, 3-O-(6"-O-acetyl) glucoside and 3-O-(6"-O-coumaroyl) glucoside anthocyanins. Actually, this could be due to the structural differences between these compounds and their association with other constituents such as the adsorption on solids (yeast, pomace) or even the modifications in their structure (formation of tannin-anthocyanin complexes) [36]. Extraction with $70 \%$ of ethanol has nevertheless been shown to improve the recovery of anthocyanins from grape by-products. This result was in accordance with several works confirming that anthocyanin yields could be improved by using high ethanol concentration solvents. Cacae et al. [37] reported that extraction of anthocyanins from black currants using aqueous ethanol increased with ethanol concentration up to $60 \%$. Another study by Lapornik et al. [38] showed that grape marc extracted with ethanol $70 \%$ had higher absolute values of anthocyanins than those extracted with water. Moreover, the anthocyanin extraction yield could also be enhanced by improving the extraction method as already shown by Howard et al. [39]. The authors studied anthocyanin contents in strawberry puree and demonstrated that strict oxygen exclusion during processing (i.e., under carbon dioxide or nitrogen) could prevent oxidative reactions.

Among the studied varieties, CAR and ALI were shown to be a rich source of anthocyanins, especially with the $70 \%$ hydro-alcoholic extract. The SYR2 also contained substantial amounts of acetylated and coumaroylated anthocyanins in EA70. 


\subsection{Antioxidant Activities Evaluation of Grape Pomace Seed and Skin Extracts}

The antioxidant potential was determined in order to select the most active grape pomace seeds and skins among the studied varieties. The assessment of antioxidant capacity has been the subject of extensive studies and arguments over the past decade. The choice of assay method is often based on speed, simplicity, ease of use and instrumentation availability. Generally, antioxidant measurements can be related either to the capacity of extracts to directly transfer hydrogen to a radical (DPPH or ABTS), to donate electrons (FRAP) or to act as competitors for peroxy radicals (ORAC test) [40]. Thus, the antioxidant capacity of each extract cannot be determined by a single method. More than one type of measurement needs to be performed to take into account the various mode of action of antioxidants $[41,42]$. In that context, in this work the free radical scavenging potential was evaluated by three spectrophotometric tests: the FRAP, $\mathrm{ABTS}^{\circ+}$ and DPPH and a spectrofluorimetric test, the ORAC test.

Concerning seed extracts, the four antioxidant analytical techniques gave the same classification both for EAQ and EA70. The highest antioxidant activities were found in SYR1 for both types of extracts. Results were correlated with previous analysis which evidenced SYR1 as having a substantial amount of flavan-3-ols, procyanidins and anthocyanins. GRE1 extract presented a low antioxidant activity as a consequence of its low phenolic contents (ORAC: $1466.4 \mu \mathrm{M}$ TE/g DW; FRAP: $0.63 \mathrm{mM} \mathrm{Fe}{ }^{2+} / \mathrm{g}$ DW, ABTS: $1203.2 \mu \mathrm{M}$ TE/g DW and DPPH: $410.8 \mathrm{TE} / \mathrm{g}$ DW in EAQ and ORAC: $1926.7 \mu \mathrm{M}$ TE/g DW; FRAP: $1.28 \mathrm{mM} \mathrm{Fe}{ }^{2+} / \mathrm{g}$ DW, ABTS: $2813.1 \mu \mathrm{M} \mathrm{TE} / \mathrm{g}$ DW and DPPH: $1277.6 \mathrm{TE} / \mathrm{g}$ DW in EA70). Antioxidant activities of EAQ and EA70 grape pomace seed extracts were showed in Table 5.

Table 5. Antioxidant activity characterisation in EAQ and EA70 grape pomace seed extracts.

\begin{tabular}{|c|c|c|c|c|c|c|}
\hline & \multicolumn{3}{|c|}{ Seeds-EAQ } & \multicolumn{3}{|c|}{ Seeds-EA70 } \\
\hline & GRE1 a & SYR1 $^{\text {a }}$ & CAR $^{a}$ & GRE1 a & SYR1 a $^{a}$ & CAR $^{a}$ \\
\hline ORAC $^{b}$ & $1466.39 \pm 29.58 \mathrm{a}$ & $2230.69 \pm 101.74 b$ & $2058.58 \pm 85.11 b$ & $1926.73 \pm 108.55 \mathrm{a}$ & $2613.98 \pm 150.86 \mathrm{a}$ & $2332.90 \pm 91.94 \mathrm{a}$ \\
\hline FRAP ${ }^{b}$ & $0.63 \pm 0.02 \mathrm{a}$ & $1.33 \pm 0.08 \mathrm{c}$ & $1.06 \pm 0.08 \mathrm{~b}$ & $1.28 \pm 0.01 \mathrm{a}$ & $1.45 \pm 0.16 \mathrm{a}$ & $1.20 \pm 0.06 \mathrm{a}$ \\
\hline $\mathbf{A B T S}^{\mathbf{b}}$ & $1203.20 \pm 24.09 a$ & $2432.62 \pm 55.95 \mathrm{c}$ & $1948.75 \pm 61.10 b$ & $2813.15 \pm 89.95 \mathrm{a}$ & $3601.20 \pm 88.59 b$ & $3495.58 \pm 66.40 \mathrm{~b}$ \\
\hline DPPH $^{\mathbf{b}}$ & $410.79 \pm 43.30 \mathrm{a}$ & $1037.12 \pm 64.04 \mathrm{~b}$ & $1050.59 \pm 30.11 b$ & $1277.59 \pm 54.69 \mathrm{a}$ & $1685.87 \pm 130.65 b$ & $1536.77 \pm 38.92 b$ \\
\hline
\end{tabular}

a GRE1, Grenache; SYR1, Syrah; CAR, Carignan. Data are expressed as the mean of triplicate \pm SD. ${ }^{\mathrm{b}}$ ORAC, ABTS and DPPH are expressed as $\mu$ mol Trolox/g DW and FRAP as mmol Fe ${ }^{2+} / \mathrm{g} \mathrm{DW}$. a, b, c; ANOVA was made to compare values obtain between varieties for the same test. Same letters indicate no significant differences between the value (Tukey's test, $p<0.05$ ).

In skins, results obtained by the different antioxidant analyses were more disparate, especially in EA70 extracts (Table 6). In aqueous extracts, the highest antioxidant activity was found in SYR1 and ALI. This observation was observed with every test and correlated well with previous results evidencing these extracts as containing high phenolic content. In EA70, different antioxidant tests did not give the same extract classification. Despite this fact, SYR1 skin extract was classified as being the first or second extract showing the highest antioxidant capacity in the four tests (ORAC: 1912.6 $\mu \mathrm{M} \mathrm{TE} / \mathrm{g} \mathrm{DW}$; FRAP: $1.52 \mathrm{mM} \mathrm{Fe}^{2+} / \mathrm{g}$ DW, ABTS: $2614.5 \mu \mathrm{M}$ TE/g DW and DPPH: $\left.1391.7 \mathrm{TE} / \mathrm{g} \mathrm{DW}\right)$.

Regression analyses (correlation coefficient $\mathrm{R}^{2}$ ) were attempted in order to correlate the results obtained with different methods. The best correlations with total phenolic contents were obtained with 
EAQ extracts both for seed and skin extracts: from $\mathrm{R}^{2}=0.87$ for DPPH to $\mathrm{R}^{2}=0.99$ for FRAP and from $\mathrm{R}^{2}=0.79$ for $\mathrm{DPPH}$ to $\mathrm{R}^{2}=0.97$ for ABTS in seed and skin extracts, respectively. Weaker correlations from $\mathrm{R}^{2}=0.43$ for ORAC to $\mathrm{R}^{2}=0.72$ for ABTS in seeds and from $\mathrm{R}^{2}=0.34$ for FRAP to $\mathrm{R}^{2}=0.63$ for ABTS in skins were observed in EA70 extracts. Positive correlations between TPC and antiradical activity using similar tests on grape seed samples and various plant samples have also been observed by other investigators [43-45]. Furthermore, this study demonstrated that correlations between grape pomace contents and antioxidant levels were higher with total values than with the specific compound concentrations quantified by HPLC. As noted in a recent publication [46], our result illustrated that antioxidant activity is more related to the total constituent levels than to the concentration of any individual compound, despite the fact that some compounds may contribute more than the others.

Table 6. Antioxidant activity characterisation in EAQ and EA70 grape pomace skin extracts.

\begin{tabular}{|c|c|c|c|c|c|c|}
\hline & \multicolumn{6}{|c|}{ Skins-EAQ } \\
\hline & GRE2 a & SYR1 a & SYR2 a & CAR $^{a}$ & MOU $^{a}$ & $\mathbf{A L I}^{\mathrm{a}}$ \\
\hline ORAC $^{\text {b }}$ & $1190.70 \pm 183.58 \mathrm{ab}$ & $1345.94 \pm 19.15 \mathrm{ab}$ & $1065.98 \pm 84.21 \mathrm{a}$ & $1077.76 \pm 60.16 \mathrm{a}$ & $1033.76 \pm 77.61 \mathrm{a}$ & $1714.62 \pm 14.77 b$ \\
\hline FRAP $^{b}$ & $0.56 \pm 0.01 \mathrm{c}$ & $0.88 \pm 0.01 \mathrm{e}$ & $0.14 \pm 0.02 \mathrm{a}$ & $0.67 \pm 0.02 \mathrm{~d}$ & $0.32 \pm 0.01 b$ & $1.13 \pm 0.00 \mathrm{f}$ \\
\hline ABTS $^{\mathbf{b}}$ & $934.12 \pm 11.9 b$ & $1427.98 \pm 54.80 \mathrm{c}$ & $668.30 \pm 29.99 a$ & $1048.83 \pm 101.57 b$ & $965.59 \pm 16.63 b$ & $1760.08 \pm 91.03 \mathrm{~d}$ \\
\hline \multirow[t]{3}{*}{ DPPH $^{b}$} & $99.45 \pm 10.82 \mathrm{a}$ & $690.29 \pm 147.01 b c$ & $263.85 \pm 71.54 \mathrm{ab}$ & $591.01 \pm 85.59 \mathrm{abc}$ & $279.43 \pm 61.65 \mathrm{ab}$ & $1057.12 \pm 45.22 \mathrm{c}$ \\
\hline & \multicolumn{6}{|c|}{ Skins-EA70 } \\
\hline & GRE2 a & SYR1 a & SYR2 a & $\mathrm{CAR}^{\mathrm{a}}$ & MOU $^{a}$ & $\mathbf{A L I}^{\mathbf{a}}$ \\
\hline ORAC $^{b}$ & $1828.26 \pm 40.37 \mathrm{bc}$ & $1912.56 \pm 6.09 b c$ & $1701.83 \pm 88.34 b c$ & $1238.38 \pm 11.09 \mathrm{a}$ & $2070.03 \pm 60.64 c$ & $1628.45 \pm 82.58 b$ \\
\hline FRAP $^{b}$ & $1.32 \pm 0.03 \mathrm{c}$ & $1.52 \pm 0.05 \mathrm{~d}$ & $0.94 \pm 0.03 \mathrm{a}$ & $1.34 \pm 0.03 \mathrm{c}$ & $1.03 \pm 0.02 \mathrm{ab}$ & $1.13 \pm 0.01 b$ \\
\hline ABTS $^{\mathbf{b}}$ & $2612.08 \pm 130.93 \mathrm{a}$ & $2614.5 \pm 10.42 \mathrm{a}$ & $2010.64 \pm 146.96 \mathrm{a}$ & $2555.92 \pm 146.04 a$ & $2674.84 \pm 187.30 \mathrm{a}$ & $1923.37 \pm 87.01 \mathrm{a}$ \\
\hline DPPH $^{b}$ & $876.96 \pm 74.32 \mathrm{a}$ & $1391.69 \pm 37.24 b c$ & $1164.91 \pm 55.55 \mathrm{ab}$ & $1075.39 \pm 46.16 \mathrm{ab}$ & $833.28 \pm 26.37 \mathrm{a}$ & $1749.31 \pm 112.65 \mathrm{c}$ \\
\hline
\end{tabular}

a GRE2, Grenache; SYR1 and SYR2, Syrah; CAR, Carignan; MOU, Mourvèdre; ALI, Alicante. Data are expressed as the mean of triplicate \pm SD. ${ }^{b}$ ORAC, ABTS and DPPH are expressed as $\mu$ mol Trolox/g DW and FRAP as mmol Fe ${ }^{2+} / \mathrm{g}$ DW. a, b, c, d, e, f; ANOVA was made to compare values obtain between varieties for the same test. Same letters indicate no significant differences between the value (Tukey's test, $p<0.05$ ).

Actually, the antioxidant activities of EAQ and EA70 followed the same trend as the phenol content of the extracts. EA70 extracts exhibited higher potential and proved to be more effective than EAQ extracts. However, due to the great diversity of polyphenols, the structure-activity relationship and bioavailability, the therapeutic efficacy of the antioxidants differs extensively $[41,42,47]$. Many reports still showed inconsistent and conflicting results using different approaches for the assessment of antioxidant capacity, making difficult the development of a universal method by which antioxidant activity can be measured accurately and quantitatively. Because of bioavailability, metabolism, biotransformation and chemical reactivity, in vitro capacity cannot be simply extrapolated [48]. Therefore, in order to evaluate the health effects of these extracts, in vivo experiments need to be performed and the effects of antioxidant may be evaluated using appropriate biomarkers in biological fluids and tissues. Nevertheless, in vitro antioxidant activity assays could be used as a pre-selection tool for the choice of grape pomace seed and skin extracts with high potential. These experiments evidenced seeds from Carignan and Syrah (SYR1) and skins from Carignan and Alicante as being the most antioxidant ones. 


\section{Experimental Section}

\subsection{Experimental Materials}

\subsubsection{Chemicals}

Deionized water was purified with a Milli-Q water system (Millipore, Bedford, MA, USA). HPLC grade methanol and ethanol were purchased from Scharlau (Sentmenat, Barcelona, Spain). The following chemicals were obtained from Sigma Aldrich (St. Louis, MO, USA): (+)-catechin, (-)-epicatechin, $\mathrm{B}_{1}$

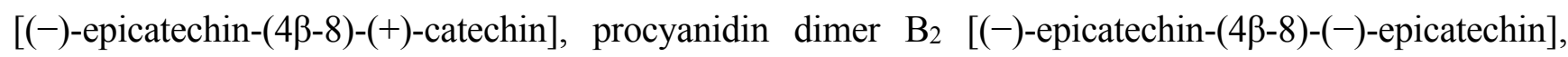
cyanidin-3-O-glucoside chloride, delphinidin-3-O-glucoside chloride, malvidin-3-O-glucoside chloride, peonidin-3-O-glucoside chloride, gallic acid, 2,2-Diphenyl-1-picrylhydrazyl (DPPH), 6-hydroxy-2,5,7,8-tetramethylchroman-2-carboxylic acid (Trolox), 2,25,7,8-tetramethylchroman-2carboxylic acid (Trolox) diammonium salt (ABTS), potassium persulfate, fluorescein, 2,2'-azobis (2-methylpropionamidine) dihydrochloride (AAPH), sodium dihydrogen phosphate dihydrate, disodium hydrogen phosphate dodecahydrate, 2,4,6-tri(2-pyridyl)-s-triazine (TPTZ), iron (III) chloride hexa-hydrate, iron (II) sulfate heptahydrate, Folin Ciocalteu's phenol $(2 \mathrm{~N})$, sodium bisulfite, sodium carbonate and formic acid. The Laboratory of Organic Chemistry and Organometallic (Université Bordeaux 1) synthesized procyanidin dimers $\mathrm{B}_{3}\left[(+)\right.$-catechin- $(4 \alpha-8)-(+)$-catechin], $\mathrm{B}_{4}[(+)$-catechin-

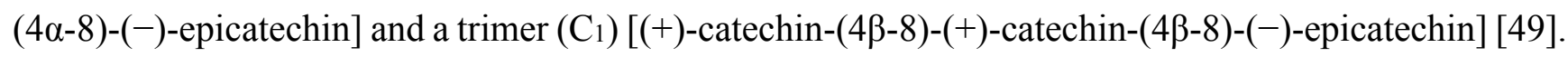

\subsubsection{Plant Materials and Sample Preparations}

In this study, samples were provided from Chateau Beaucastel, located in the Rhône Valley area. Grapes at maturity from the 2010 vintage underwent vinification processes after which their derived wines were used for commercial purpose. Grape pomaces were collected after these operations. Study was carried out using grape pomaces from $V$. vinifera L. cv. Grenache (from two different parcels [GRE1 and GRE2]), Syrah (from two different parcels [SYR1 and SYR2]), Carignan (CAR), Mourvèdre (MOU) and Alicante (ALI). Seeds were separated from skins using a mechanic separator and both were frozen at $-20^{\circ} \mathrm{C}$ prior to analysis. According to previous study ([50]), samples were selected on the basis of their high content of polyphenols. One hundred grams of GRE1, SYR1 and CAR seeds and GRE2, SYR1, SYR2, CAR, MOU and ALI skins were extracted in triplicate using $350 \mathrm{~mL}$ of distilled water for $1 \mathrm{~h}$ under magnetic agitation at $50{ }^{\circ} \mathrm{C}$. In parallel, under the same conditions, these samples were also extracted using a $70 \%$ hydro/alcoholic $(70: 30, \mathrm{v} / \mathrm{v})$ solution. The centrifugal supernatants were evaporated in vacuo at $30{ }^{\circ} \mathrm{C}$ and lyophilized to obtain two types of samples: an aqueous sample (EAQ) and a 70\% hydro-alcoholic sample (EA70) for each variety and part.

\subsection{Total Phenolics, Tannins and Anthocyanins}

Total polyphenol, tannin and anthocyanin contents of grape pomace skin and seed extracts were determined. Crude extracts were solubilized in water/ethanol (90:10, v/v; $\mathrm{pH} 3.5$ with tartaric acid) at appropriate concentrations. Total phenol content (TPC) was determined by the Folin-Ciocalteu assay [51] and the data expressed as mg of gallic acid equivalents (GAE) per g dry weight. Total tannin 
content was measured by acidic hydrolysis using the method of Ribereau-Gayon and Stonestreet [52]. Anthocyanin content was determined by the $\mathrm{SO}_{2}$ bleaching procedure [53].

\subsection{HPLC Analysis of Monomeric/Oligomeric Tannins}

Monomeric/oligomeric tannin extracts were solubilized in a methanol/water solution $(50: 50, \mathrm{v} / \mathrm{v})$ at appropriate concentrations and analyses were carried out according to the method of Silva et al. 2011 [54].

\subsection{HPLC Analysis of Anthocyanins}

Grape pomace extracts were solubilized in methanol/water $(50: 50, \mathrm{v} / \mathrm{v})$ containing $1 \%$ formic acid. Analysis were carried out using a Surveyor HPLC with sampler cooler maintained at $4{ }^{\circ} \mathrm{C}$, a PDA detector scanning from 200 to $600 \mathrm{~nm}$ and a LCQ Advantage ion trap mass spectrometer with a split volume set at $0.2 \mathrm{~mL} / \mathrm{min}$ and ESI operating in full-scan positive mode scanning from $\mathrm{m} / \mathrm{z} 200$ to 1000 . Separation was performed on a $250 \times 4.6 \mathrm{~mm}$ i.d. $4 \mu \mathrm{m}$ Synergi RP-Max column (Phenomenex, Macclesfield, UK) maintained at $40{ }^{\circ} \mathrm{C}$ in a column oven. The mobile phase pumped at $1 \mathrm{~mL} / \mathrm{min}$ comprised a $65 \mathrm{~min}, 10 \%-45 \%$ gradient of methanol in water with both solvents containing $1 \%$ formic acid. The injection volume was $10 \mu \mathrm{L}$. Peak detection and quantification were monitored at $520 \mathrm{~nm}$ and performed by comparison with available standards or confirmed by mass spectrometry in full-scan positive ionization, data dependent $\mathrm{MS}^{2}$. Anthocyanins were quantified as malvidin-3-O-glucoside equivalents and expressed in mg per dry weight of seed or skin $\pm \operatorname{SE}(n=3)$.

\subsection{Antioxidant Assays}

\subsubsection{Oxygen Radical Absorbance Capacity (ORAC) Assay}

The ORAC assay was applied according to the method of $\mathrm{Ou}$ et al. [55] as modified by Dávalos et al. [56]. The procedure was carried out using an automated plate reader (BMG LABTECH, Ortenberg, Germany) equipped with a fluorescence detector set at excitation and emission wavelengths of $485 \mathrm{~nm}$ and $530 \mathrm{~nm}$ respectively. Analyses were conducted in a phosphate buffer (pH 7.4, $75 \mathrm{mM}$ ). Peroxyl radical were generated using AAPH $(40 \mathrm{mM})$ and fluorescein $(117 \mathrm{nM})$ was used as the substrate. Readings were taken every minute for $90 \mathrm{~min}$ at $37^{\circ} \mathrm{C}$. The area under the curve (AUC) was calculated for each sample by integrating the relative fluorescence curve. The net AUC was calculated by subtracting the AUC of the blank. The final ORAC values were determined by linear regression equation of Trolox concentrations and are expressed as $\mu \mathrm{M}$ Trolox equivalents/g dry weights.

\subsubsection{Ferric Reducing Antioxidant Potential Assay (FRAP)}

FRAP assay was performed based on the method of Benzie and Strain [57] using an automated plate reader set at $593 \mathrm{~nm}$. FRAP reagent were prepared daily by mixing 10 volumes of $300 \mathrm{mM}$ soduim acetate buffer ( $\mathrm{pH}$ 3.6) with 1 volume of $10 \mathrm{mM}$ TPTZ solution and 1 volume $20 \mathrm{mM}$ ferric chloride. A standard curve was prepared using various concentrations of $\mathrm{FeSO}_{4} \times 7 \mathrm{H}_{2} \mathrm{O}$. Samples $(40 \mu \mathrm{L})$ were allowed to react with FRAP reagent $(300 \mu \mathrm{L})$ for 4 min in dark condition. Blank values were subtracted 
from samples and standards values then difference were used to calculate the FRAP value. Results were expressed as $\mu \mathrm{M} \mathrm{Fe}^{2+} / \mathrm{g}$ of dry skin and seed weights.

\subsubsection{ABTS Assay}

The ABTS assay was performed as described by Re et al. [58]. ABTS radical cation solution was prepared by mixing $2.45 \mathrm{mM}$ of potassium persulfate and ABTS (7 $\mathrm{mM}$ in deionized water) following by $12-16 \mathrm{~h}$ incubation in the dark at room temperature. Before use, the $\mathrm{ABTS}^{+\bullet}$ solution was diluted with deionized water to an absorbance of $0.7 \pm 0.02$ at $734 \mathrm{~nm}$ using a Jenway-6305 UV-vis spectrophotometer (Jenway, Staffordshire, UK). Samples $(100 \mu \mathrm{L})$ were allowed to react with $2 \mathrm{~mL}$ of $\mathrm{ABTS}^{+\bullet}$ solution for $10 \mathrm{~min}$. Blank values were subtracted from samples and standard values and a linear regression for the Trolox standards were constructed. Results were expressed as $\mu \mathrm{M}$ Trolox equivalents/g dry weights.

\subsubsection{DPPH Assay}

This method was used according to Brand-Williams et al. [59] modified by Miliauskas et al. [60]. Samples $(100 \mu \mathrm{L})$ were allowed to react with $2 \mathrm{~mL}$ of daily prepared $\mathrm{DPPH}^{\bullet}$ solution $\left(6 \times 10^{-5} \mathrm{M}\right.$, dissolve in methanol) for $20 \mathrm{~min}$ at room temperature. The absorbance of the resulting solution was measured at $515 \mathrm{~nm}$. Blank values were subtracted from samples and standard values. A linear regression for the Trolox standards was constructed. Results were expressed as $\mu \mathrm{M}$ Trolox equivalents $/ \mathrm{g}$ dry weights.

\subsection{Statistical Analysis}

All measurements were performed in triplicate. Results are expressed as means \pm standard deviation (SD). One-way ANOVA was performed to test the effects of variation factors (different samples) on each variable (TPC, total tannins, total anthocyanins, phenol concentrations etc.). If significant effects were found at a 95\% confidence interval, ANOVA was followed by a Tukey's HSD post hoc test to identify differences among groups. These analyses were performed using Statistica V.7 Software (Statsoft Inc., Tulsa, OK, USA).

\section{Conclusions}

This study showed that grape pomace seed and skin extracts still contained appreciable amounts of flavan-3-ols and anthocyanins, despite extraction during vinification. Overall, extraction with 70\% aqueous ethanol was shown to optimize the recovery of flavan-3-ols and anthocyanins from the grape by-products. Indeed, by using a high alcohol level for the extraction of grape pomaces, less soluble and more stable compounds can be released while more soluble and less stable ones had already been extracted. Quantitative and qualitative distribution of polyphenols in grape pomaces showed significant differences across varieties. This investigation evidenced that seeds from Carignan and Syrah and skins from Carignan and Alicante as containing high phenolic contents and antioxidant activity. This information is of significant importance for the selection of suitable extracts which could be further used in cosmetics or anti-ageing products. 


\section{Acknowledgments}

The authors gratefully thank Mr Pierre Perrin (Perrin et Fils, Chateau Beaucastel, Châteauneuf-du-Pape) for providing the Mediterranean pomaces and financial support for Isabelle Ky's postgraduate research.

\section{Author Contributions}

IKY carried out the experiments and prepared the manuscript. PLT revised and approved the final version of the manuscript.

\section{Conflicts of Interest}

The authors declare no conflict of interest.

\section{References}

1. Forman, H.J.; Maiorino, M.; Ursini, F. Signaling functions of reactive oxygen species. Biochemistry 2010, 49, 835-842.

2. Mimić-Oka, J.; Simić, D.V.; Simić, T.P. Free radicals in cardiovascular diseases. Facta Univ. Ser. Med. Biol. 1999, 6, 11-22.

3. Mennen, L.I.; Sapinho, D.; de Bree, A.; Arnault, N.; Bertrais, S.; Galan, P.; Hercberg, S. Consumption of foods rich in flavonoids is related to a decreased cardiovascular risk in apparently healthy french women. J. Nutr. 2004, 134, 923-926.

4. He, F.J.; Nowson, C.A.; Lucas, M.; MacGregor, G.A. Increased consumption of fruit and vegetables is related to a reduced risk of coronary heart disease: Meta-analysis of cohort studies. J. Hum. Hypertens. 2007, 21, 717-728.

5. Alvarez-Suarez, J.M.; Giampieri, F.; Tulipani, S.; Casoli, T.; Di Stefano, G.; González-Paramás, A.M.; Santos-Buelga, C.; Busco, F.; Quiles, J.L.; Cordero, M.D.; et al. One-month strawberry-rich anthocyanin supplementation ameliorates cardiovascular risk, oxidative stress markers and platelet activation in humans. J. Nutr. Biochem. 2014, 25, 289-294.

6. Dröge, W. Free radicals in the physiological control of cell function. Physiol. Rev. 2002, 82, 47-95.

7. Halliwell, B. Free radicals, antioxidants, and human disease: Curiosity, cause, or consequence? Lancet 1994, 344, 721-724.

8. Ky, I.; Crozier, A.; Cros, G.; Teissedre, P.-L. Polyphenols composition of wine and grape sub-products and potential effects on chronic diseases. Nutr. Aging 2014, 2, 165-177.

9. OIV. World statistics. In Proceedings of the 9th General Assembly of the OIV, Porto, Portugal, 20-27 June 2011; OIV: Paris, France, 2011.

10. Llobera, A.; Cañellas, J. Dietary fibre content and antioxidant activity of Manto Negro red grape (Vitis vinifera): pomace and stem. Food Chem. 2007, 101, 659-666.

11. Laufenberg, G.; Kunz, B.; Nystroem, M. Transformation of vegetable waste into value added products: (A) the upgrading concept; (B) practical implementations. Bioresour. Technol. 2003, 87, 167-198. 
12. Ruberto, G.; Renda, A.; Daquino, C.; Amico, V.; Spatafora, C.; Tringali, C.; Tommasi, N.D. Polyphenol constituents and antioxidant activity of grape pomace extracts from five Sicilian red grape cultivars. Food Chem. 2007, 100, 203-210.

13. Kammerer, D.; Claus, A.; Carle, R.; Schieber, A. Polyphenol screening of pomace from red and white grape varieties (Vitis vinifera L.) by HPLC-DAD-MS/MS. J. Agric. Food Chem. 2004, 52, 4360-4367.

14. Alonso, Á.M.; Guillén, D.A.; Barroso, C.G.; Puertas, B.; García, A. Determination of antioxidant activity of wine byproducts and its correlation with polyphenolic content. J. Agric. Food Chem. 2002, 50, 5832-5836.

15. Xu, Y.; Simon, J.E.; Welch, C.; Wightman, J.D.; Ferruzzi, M.G.; Ho, L.; Passinetti, G.M.; Wu, Q. Survey of polyphenol constituents in grapes and grape-derived products. J. Agric. Food Chem. 2011, 59, 10586-10593.

16. Lafka, T.I.; Sinanoglou, V.; Lazos, E.S. On the extraction and antioxidant activity of phenolic compounds from winery wastes. Food Chem. 2007, 104, 1206-1214.

17. Sáyago-Ayerdi, S.G.; Brenes, A.; Viveros, A.; Goñi, I. Antioxidative effect of dietary grape pomace concentrate on lipid oxidation of chilled and long-term frozen stored chicken patties. Meat Sci. 2009, 83, 528-533.

18. Ayed, N.; Yu, H.L.; Lacroix, M. Improvement of anthocyanin yield and shelf-life extension of grape pomace by gamma irradiation. Food Res. Int. 1999, 32, 539-543.

19. Metivier, R.P.; Francis, F.J.; Clydesdale, F.M. Solvent extraction of anthocyanins from wine pomace. J. Food Sci. 1980, 45, 1099-1100.

20. Ross, C.F.; Hoye, J.C.; Fernandez-Plotka, V.C. Influence of heating on the polyphenolic content and antioxidant activity of grape seed flour. J. Food Sci. 2011, 76, C884-C890.

21. Saura-Calixto, F. Antioxidant dietary fiber product: A new concept and a potential food ingredient. J. Agric. Food Chem. 1998, 46, 4303-4306.

22. González-Paramás, A.M.; Esteban-Ruano, S.; Santos-Buelga, C.; de Pascual-Teresa, S.; Rivas-Gonzalo, J.C. Flavanol content and antioxidant activity in winery byproducts. J. Agric. Food Chem. 2003, 52, 234-238.

23. Shrikhande, A.J. Wine by-products with health benefits. Food Res. Int. 2000, 33, 469-474.

24. Morel-Salmi, C.; Souquet, J.-M.; Bes, M.; Cheynier, V. Effect of flash release treatment on phenolic extraction and wine composition. J. Agric. Food Chem. 2006, 54, 4270-4276.

25. Chira, K.; Lorrain, B.; Ky, I.; Teissedre, P.L. Tannin composition of cabernet-sauvignon and merlot grapes from the bordeaux area for different vintages (2006 to 2009) and comparison to tannin profile of five 2009 vintage mediterranean grapes varieties. Molecules 2011, 16, 1519-1532.

26. Jackson, R.S. Chapter 2: Grape Species and Varieties. In Wine Science, 2nd ed.; Jackson, R.S., Ed.; Academic Press: San Diego, CA, USA, 2000; pp. 13-44.

27. Freitas, V.A.P.D.; Glories, Y. Concentration and compositional changes of procyanidins in grape seeds and skin of white Vitis vinífera varieties. J. Sci. Food Agric. 1999, 79, 1601-1606.

28. Monagas, M.; Gómez-Cordovés, C.; Bartolomé, B.; Laureano, O.; Ricardo da Silva, J.M. Monomeric, oligomeric, and polymeric flavan-3-ol composition of wines and grapes from Vitis vinifera L. Cv. Graciano, Tempranillo, and Cabernet Sauvignon. J. Agric. Food Chem. 2003, $51,6475-6481$. 
29. Kobriger, J.M.; Kliewer, W.M.; Lagier, S.T. Effects of wind on water relations of several grapevine cultivars. Am. J. Enol. Vitic. 1984, 35, 164-169.

30. Muniz, J.E.; Wample, R.L.; Loescher, W.H. Cultivar differences in response to low temperatures in Vitis vinifera callus in vitro. Am. J. Enol. Vitic. 1991, 42, 341-346.

31. Fournand, D.; Vicens, A.; Sidhoum, L.; Souquet, J.-M.; Moutounet, M.; Cheynier, V. Accumulation and extractability of grape skin tannins and anthocyanins at different advanced physiological stages. J. Agric. Food Chem. 2006, 54, 7331-7338.

32. Vergara-Salinas, J.R.; Bulnes, P.; Zúñiga, M.C.; Pérez-Jiménez, J.; Torres, J.L.; Mateos-Martín, M.L.; Agosin, E.; Pérez-Correa, J.R. Effect of pressurized hot water extraction on antioxidants from grape pomace before and after enological fermentation. J. Agric. Food Chem. 2013, 61, 6929-6936.

33. Acevedo De la Cruz, A.; Hilbert, G.; Rivière, C.; Mengin, V.; Ollat, N.; Bordenave, L.; Decroocq, S.; Delaunay, J.-C.; Delrot, S.; Mérillon, J.-M.; et al. Anthocyanin identification and composition of wild Vitis spp. accessions by using LC-MS and LC-NMR. Anal. Chim. Acta 2012, 732, 145-152.

34. Mazza, G.; Francis, F.J. Anthocyanins in grapes and grape products. Crit. Rev. Food Sci. Nutr. 1995, 35, 341-371.

35. Ribéreau-Gayon, P. Recherches sur les Anthocyanes des Végétaux: Application au Genre Vitis. Ph.D. Thesis, Université de Paris, Paris, France, 1959.

36. Ribéreau-Gayon, P.; Branco, J.M.; Rychlewski, C. Handbook of Enology. The Chemistry of Wine. Stabilization and Treatments; John Wiley \& Sons Ltd: Chichester, UK, 2006; Volume 2.

37. Cacace, J.E.; Mazza, G. Optimization of extraction of anthocyanins from black currants with aqueous ethanol. J. Food Sci. 2003, 68, 240-248.

38. Lapornik, B.; Prošek, M.; Golc Wondra, A. Comparison of extracts prepared from plant by-products using different solvents and extraction time. J. Food Eng. 2005, 71, 214-222.

39. Howard, L.R.; Brownmiller, C.; Prior, R.L. Improved color and anthocyanin retention in strawberry puree by oxygen exclusion. J. Berry Res. 2014, 4, 107-116.

40. Roginsky, V.; Lissi, E.A. Review of methods to determine chain-breaking antioxidant activity in food. Food Chem. 2005, 92, 235-254.

41. Huang, D.; Ou, B.; Prior, R.L. The chemistry behind antioxidant capacity assays. J. Agric. Food Chem. 2005, 53, 1841-1856.

42. Niki, E. Antioxidant capacity: Which capacity and how to assess it? J. Berry Res. 2011, 1, 169-176.

43. Bozan, B.; Tosun, G.; Özcan, D. Study of polyphenol content in the seeds of red grape (Vitis vinifera L.) varieties cultivated in Turkey and their antiradical activity. Food Chem. 2008, 109, 426-430.

44. Dudonné, S.; Vitrac, X.; Coutière, P.; Woillez, M.; Mérillon, J.M. Comparative study of antioxidant properties and total phenolic content of 30 plant extracts of industrial interest using DPPH, ABTS, FRAP, SOD, and ORAC assays. J. Agric. Food Chem. 2009, 57, 1768-1774.

45. Ma, X.; Wu, H.; Liu, L.; Yao, Q.; Wang, S.; Zhan, R.; Xing, S.; Zhou, Y. Polyphenolic compounds and antioxidant properties in mango fruits. Sci. Hortic. 2011, 129, 102-107.

46. Rockenbach, I.I.; Gonzaga, L.V.; Rizelio, V.M.; de Souza Schmidt Gonçalves, A.E.; Genovese, M.I.; Fett, R. Phenolic compounds and antioxidant activity of seed and skin extracts of red grape (Vitis vinifera and Vitis labrusca) pomace from Brazilian winemaking. Food Res. Int. 2011, 44, 897-901. 
47. Takashima, M.; Horie, M.; Shichiri, M.; Hagihara, Y.; Yoshida, Y.; Niki, E. Assessment of antioxidant capacity for scavenging free radicals in vitro: A rational basis and practical application. Free Radic. Biol. Med. 2012, 52, 1242-1252.

48. Chiva-Blanch, G.; Visioli, F. Polyphenols and health: Moving beyond antioxidants. J. Berry Res. 2012, 2, 63-71.

49. Tarascou, I.; Barathieu, K.; André, Y.; Pianet, I.; Dufourc, E.J.; Fouquet, E. An improved synthesis of procyanidin dimers: Regio- and stereocontrol of the interflavan bond. Eur. J. Org. Chem. 2006, 5367-5377.

50. Ky, I.; Lorrain, B.; Kolbas, N.; Crozier, A.; Teissedre, P.L. Wine by-products: Phenolic characterization and antioxidant activity evaluation of grapes and grape pomaces from six different French grape varieties. Molecules 2014, 19, 482-506.

51. Singleton, V.L.; Rossi, J.A. Colorimetry of total phenolics with phosphomolybdic-phosphotungstic acid reagents. Am. J. Enol. Vitic. 1965, 16, 144-158.

52. Ribéreau Gayon, P.; Stonestreet, E. Le dosage des tanins dans le vin rouge et détermination de leur structure. Chim. Anal. 1966, 188-196.

53. Ribéreau Gayon, P.; Stonestreet, E. Le dosage des anthocyanes dans le vin rouge. Bull. Soc. Chim. Fr. 1965, 2649-2652.

54. Silva, M.A.; Ky, I.; Jourdes, M.; Teissedre, P.L. Rapid and simple method for the quantification of flavan-3-ols in wine. Eur. Food Res. Technol. 2012, 234, 361-365.

55. Ou, B.; Hampsch-Woodill, M.; Prior, R.L. Development and validation of an improved oxygen radical absorbance capacity assay using fluorescein as the fluorescent probe. J. Agric. Food Chem. 2001, 49, 4619-4626.

56. Dávalos, A.; Gómez-Cordovés, C.; Bartolomé, B. Extending applicability of the oxygen radical absorbance capacity (ORAC-Fluorescein) assay. J. Agric. Food Chem. 2004, 52, $48-54$.

57. Benzie, I.F.F.; Strain, J.J. The ferric reducing ability of plasma (FRAP) as a measure of "antioxidant power": The FRAP assay. Anal. Biochem. 1996, 239, 70-76.

58. Re, R.; Pellegrini, N.; Proteggente, A.; Pannala, A.; Yang, M.; Rice-Evans, C. Antioxidant activity applying an improved ABTS radical cation decolorization assay. Free Radic. Biol. Med. 1999, 26, 1231-1237.

59. Brand-Williams, W.; Cuvelier, M.E.; Berset, C. Use of a free radical method to evaluate antioxidant activity. LWT-Food Sci. Technol. 1995, 28, 25-30.

60. Miliauskas, G.; Venskutonis, P.R.; Van Beek, T.A. Screening of radical scavenging activity of some medicinal and aromatic plant extracts. Food Chem. 2004, 85, 231-237.

Sample Availability: Samples are available from the authors.

(C) 2015 by the authors; licensee MDPI, Basel, Switzerland. This article is an open access article distributed under the terms and conditions of the Creative Commons Attribution license (http://creativecommons.org/licenses/by/4.0/). 